\title{
Lagrangian decomposition approach to scheduling large-scale refinery operations
}

\author{
Q2 Nikisha K. Shah, Marianthi G. Ierapetritou* \\ Chemical and Biochemical Engineering Department, Rutgers University, NJ, USA
}

\section{A R T I C L E I N F O}

\section{Article history:}

Received 2 December 2014

Received in revised form 14 April 2015

Accepted 22 April 2015

Available online $\mathrm{xxx}$

\section{Keywords:}

Refinery operations scheduling

Blend scheduling

Lagrangian decomposition

Mixed-integer linear programming

\begin{abstract}
A B S T R A C T
This work focuses on the scheduling of refinery operations from crude oil processing to the blending and dispatch of finished products. A new algorithm for Lagrangian decomposition (LD) is proposed and applied to realistic large scale refinery scheduling problem to evaluate its efficiency. A novel strategy is presented to formulate restricted relaxed sub-problems based on the solution of the Lagrangian relaxed sub-problems that take into consideration the continuous process characteristic of the refinery. This new algorithm, referred to as restricted Lagrangian decomposition algorithm, the best lower bound is obtained amongst the restricted-relaxed sub-problems and relaxed sub-problems in each iteration. The goal of the decomposition is to produce better solutions for those integrated scheduling problems that cannot be solved in reasonable computation times. The application of the proposed algorithm results in substantial reduction in CPU solution time, duality gap, and the total number of iterations compared to classical LD.
\end{abstract} (c) 2015 Published by Elsevier Ltd.

\footnotetext{
* Corresponding author. Tel.: +1 8484452971.

E-mail address: marianth@soemail.rutgers.edu (M.G. Ierapetritou).
} 


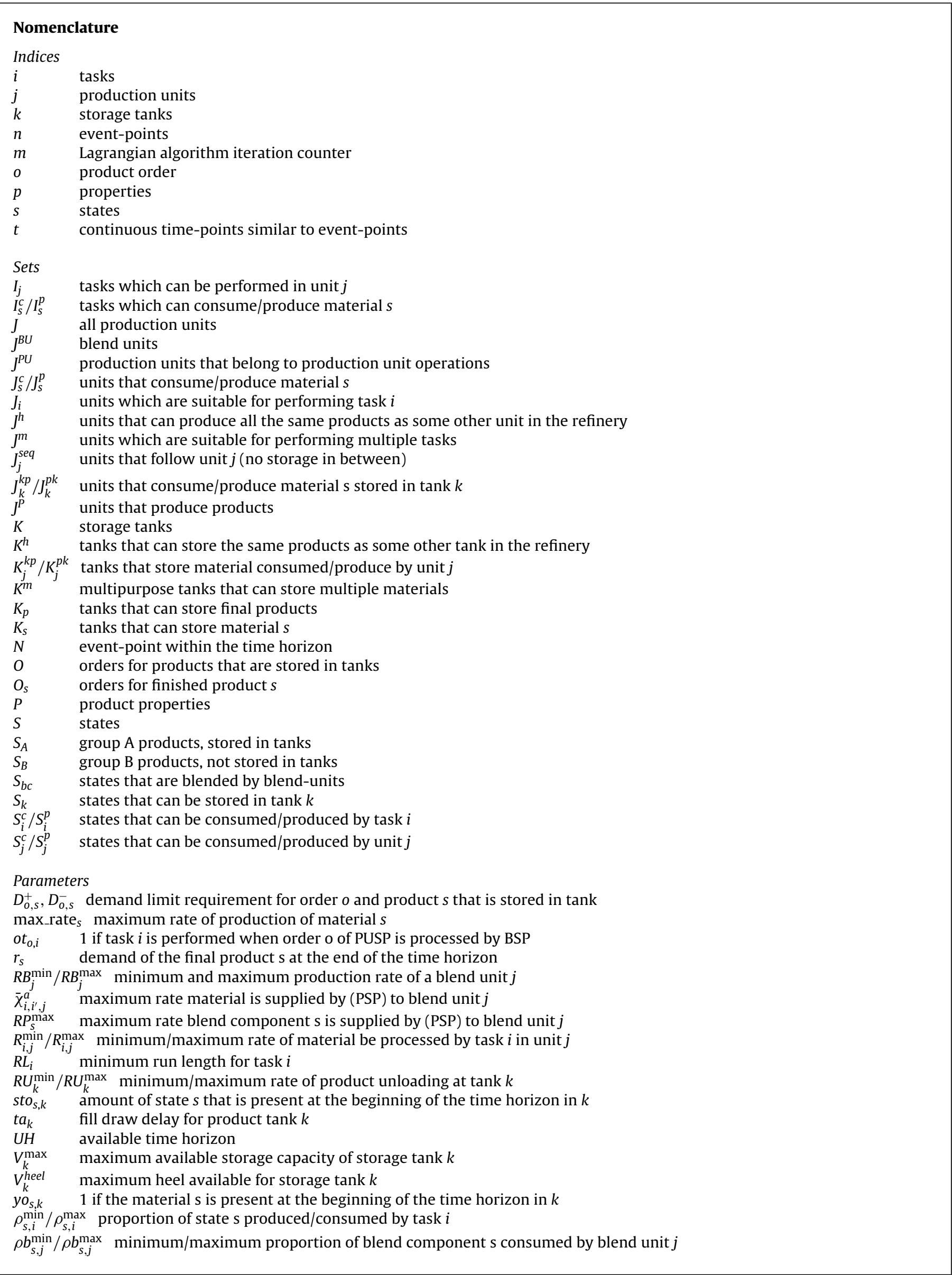




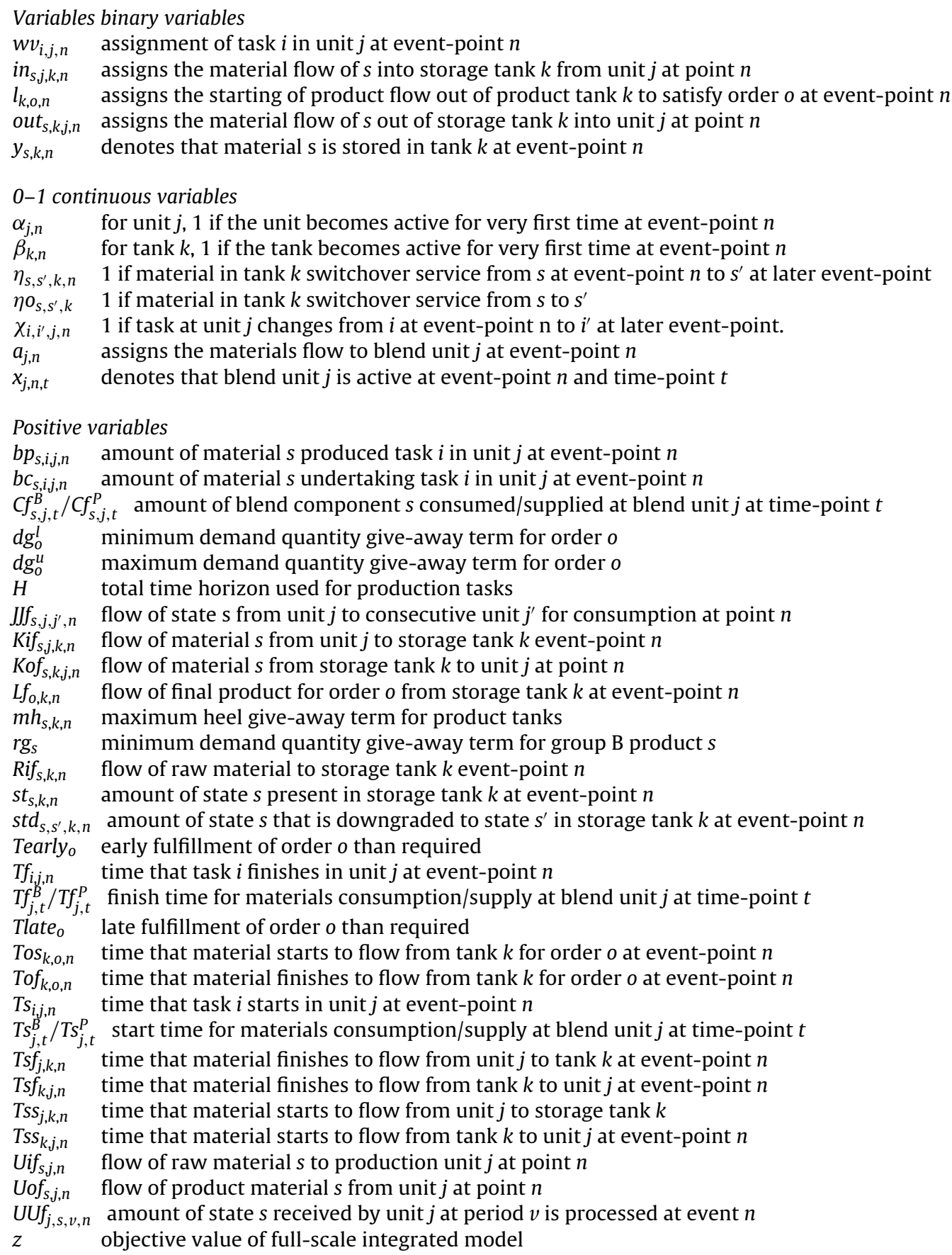

proposed by Jia and Ierapetritou (2004), Joly et al. (2002), and Shah et al. (2009). Castillo and Mahalec (2014a,b), Castro and Grossmann (2014), Glismann and Gruhn (2001), Jia and Ierapetritou (2003), Kelly (2006), Kolodziej et al. (2013), Li and Karimi (2011), Mendez et al. (2006), and Neiro et al. (2014) have addressed finished product blending and distribution scheduling problem. Kelly (2006) emphasized the importance of logistics details in refinery blending and delivery problem and proposed a decomposition of the blend scheduling problem into two sub-problems, logistics and quality. The logistics sub-problem considers only the quantity and logistics variables and the problem constraints whereas the quality sub-problem considers product specifications, quantity constraints and bounds. Their work is based upon discrete time formulation, and they include many logistics details such as, lower-up time, sequence dependent changeovers, and fill-draw-delay. They observed that incorporating logistics details into scheduling problem can yield substantial improvements in efficiency and productivity. However, many of the logistics rules associated with blending are not considered due to the complexity of a resulting blending model. To deal with complexity of the blending problem, several simplifications are made to the real problem, such as considering fixed recipes instead of variable recipes (Jia and Ierapetritou, 2003) and constant flowrates for blend components and products (Mendez et al., 2006). 


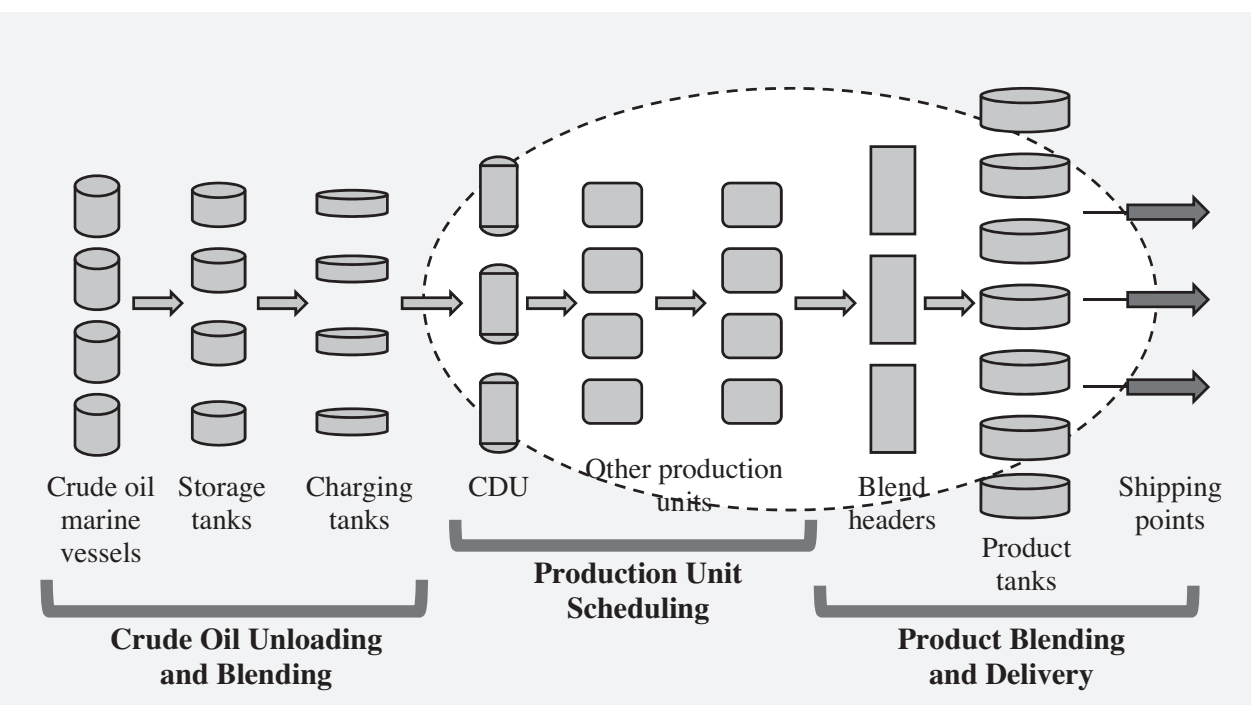

Fig. 1. Schematic of a standard refinery system.

Usually blend component tanks are present when the blending and delivery sub-problem is addressed without simultaneously addressing production unit scheduling problem. However, when blend components tanks are not available, blend units are directly connected to the upstream production units (Fig. 1). In order to comply with the finished blend product properties specifications, blend recipe needs be determined by considering the production constraints of upstream processes so that the blend components production and consumption rate is consistent across two scheduling decision levels. These interdependences of the blending operations and production unit scheduling operations require an integrated approach to the refinery scheduling. Scant attention has been paid in the literature in addressing the short-term scheduling of an integrated production unit operations and finished product blending and delivery problem. It is imperative to model an integrated production unit and finished product blending-delivery scheduling problem to address the issue of online blend units. The resulting comprehensive model is complex, difficult to solve due to non-linear blend product properties, logistics constraints such as set-ups, and changeovers. Moro et al. (1998) proposed a planning model for refinery diesel production where the emphasis is on blend relations. Pinto et al. (2000) proposed a planning and scheduling model for refinery operations. They presented the formulation based on discretization of time for production and distribution scheduling and their model included the features such as sequence dependent transition cost of products within oil pipeline. Luo and Rong (2007) developed a two decisions levels scheduling model for overall refinery. The upper level optimization model is based on discrete time formulation and it is used to determine sequencing and timing of operations modes and to decide the quantities of materials produced/consumed at each operations mode. The upper level decisions level uses aggregated tanks storage capacity whereas the lower level uses heuristics to obtain a detailed schedule. They consider multi-purpose product tanks via an iterative procedure that allows to readjust aggregated tank capacity at lower level by changing multipurpose tank service mode and then to recalculate corresponding optimal solution at upper level. The logistics details are ensured through heuristics at the lower level.

In our previous work, Shah and Ierapetritou (2011) have proposed a model based on continuous-time representation and the notion of event-points for the simultaneous scheduling of production unit operations and end-product blending and delivery operations. The model incorporates quantity, quality, and logistics decisions related to real-life refinery operations. These involve minimum run-length requirements, fill-draw-delay, one-flow out of blender, sequence dependent switchovers, maximum heel quantity, and downgrading of better quality product to lower quality. A set of valid inequalities that improves the computational performance of the model significantly are proposed. However, even with the inclusion of valid inequalities in the scheduling model, the computational expense required to reach an optimal solution is still considerably high for the real life refinery applications. Large-scale scheduling problems arise frequently in oil-refineries where the main objective is to assign a large number of tasks to the processing units over a specific time horizon such that the demand of each product is satisfied before its due date while minimizing the production cost or maximizing the total profit. The complexity arises from the large number of product demand orders, quality specifications for each finished blend products, and limited number of available resources to reach production goals. Furthermore, large-scale problems are computationally intractable in the sense that it is difficult to obtain "good", not necessary optimal, solutions. Hence, there is a need to employ different decomposition approach to enable the solution of large-scale problems in a reasonable computational time.

In this work, the integrated refinery operations scheduling problem is addressed using Lagrangian decomposition. Lagrangian decomposition (LD) is based upon relaxing the complicating constraints so that the problem is decomposed into smaller problems. The complicating constraints are replaced with a penalty term in the objective function involving the amount of violation of the constraints and their dual variables known as Lagrange multipliers according to Fisher (1981) and Held and Karp (1970). Lagrangian relaxation method has been successfully applied to various problems, such as scheduling (Adhya et al., 1999; Ghaddar et al., 2014; Luh and Hoitomt, 1993; Wu and Ierapetritou, 2003), planning (Graves, 1982; Gupta and Maranas, 1999; Tang and Jiang, 2009), and integrated planning and scheduling (Calfa et al., 2013; Li and Ierapetritou, 2010; Mouret et al., 2011; Shah and Ierapetritou, 2012). Lagrangian relaxation is also used to calculate lower bound in outer approximation algorithm (Karuppiah et al., 2008) and branch and bound approach (Holmberg and Hellstrand, 1998; Holmberg and Yuan, 2000).

The paper is organized in the following way. Section 2 describes the integrated refinery operations problem. Section 3 presents solution strategy for the integrated problem, whereas Section 4 describes Lagrangian decomposition (LD) algorithm, proposes novel heuristic 
approach to construct restricted Lagrangian sub-problems to attain tighter lower bounds and to obtain a feasible solution for an upper bounding problem. Section 5 provides results, and the paper concludes with Section 6.

\section{Refinery operations scheduling problem}

The integrated refinery operations scheduling model (IP) proposed in our previous work serves as a benchmark for the proposed decomposition algorithm. The formulation for the refinery operations scheduling problem is based on the continuous time representation and an notion of a unit-specific event-points first introduced by lerapetritou and Floudas (1998a,b) and Ierapetritou et al. (1999). Statetask network representation introduced by Kondili et al. (1993) is used in formulating the problem. The refinery system considered here consists of raw material storage tanks, production units, blending units, intermediate tanks, and final product tanks. For simplicity, the final products are organized into two sets: (a) set $S_{A}$ is comprised of blend products that are stored in tanks and (b) set $S_{B}$ comprised of products that are not stored in tanks but are supplied to the market directly from production units to be used as feedstock in chemical industries. The use of term "demand order" is limited to the products that belong to the first group and each demand order corresponds to only one kind of product since each multiproduct order can be decomposed into several single product orders. Furthermore, demand order set is arranged according to the ascending due date start time.

We present a brief overview of the integrated model in this work and the available information is (a) production and consumption recipe bounds of materials for each production and blend units; (b) upper and lower bounds on production flow-rates; (c) maximum and minimum inventory capacities for tanks, identity of materials that can be store in each tanks, initial holdup in each tanks; and maximum heel quantity for multipurpose product tanks (d) maximum lift-rate from finished blend product tanks; (e) product quality limitations (f) minimum demands for middle distillate products; ( $g$ ) maximum and minimum demand orders for finished blend products and delivery window each demand order; (h) minimum run-length for production and blend units; (i) available scheduling horizon (j) coefficients for calculating various costs involved.

Due to the space limitation, aggregated version of the refinery operations scheduling model (IP) is presented here and the reader is directed to our previous work (Shah and Ierapetritou, 2011) for complete model.

$$
\begin{aligned}
z & =\sum_{i, j \in J_{i}, n} c_{i, j}^{1} w v_{i, j, n}+\sum_{k \in K_{p}, o, n} c_{k}^{2} l_{k, o, n}+\sum_{s, k \in K_{s}, n} c_{k}^{3} s t_{s, k, n}+\sum_{j \in J^{h}, n} c_{j}^{4} \alpha_{j, n}+\sum_{k \in K^{h}, n} c_{k}^{5} \beta_{k, n}+\sum_{j \in J^{m}, i \in I_{j}, i^{\prime} \in I_{j}, i^{\prime} \neq i, n} c_{i, i^{\prime}}^{6} \chi_{i, i^{\prime}, j, n} \\
& +\sum_{k \in K^{m}, s \in S_{k}, s^{\prime} \in S_{k}, s \neq s^{\prime}} c_{s, s^{\prime}}^{7} \eta o_{s, s^{\prime}, k}+\sum_{k \in K^{m}, s \in S_{k}, s^{\prime} \in S_{k}, s \neq s^{\prime}, n} c_{s, s^{\prime}}^{8} \eta_{s, s^{\prime}, k, n}+\sum_{k \in K^{m}}^{9} s t d_{s, s^{\prime}, k, n}+\sum_{k \in K^{m}, s \in S_{k}, s^{\prime} \in S_{k}, s \neq s^{\prime}, n} c_{s, k}^{10} m h_{s, k, n} \\
& +\sum_{o \in O} c_{o}^{11} d g_{o}^{l}+\sum_{o \in O} c_{o}^{12} d g_{o}^{u}+\sum_{s \in S_{B}} c_{s}^{13} r g_{s}+\sum_{o \in O} c_{o}^{14} \text { Tearly }_{o}+\sum_{o \in O} c_{o}^{15} \operatorname{Tlate}_{o}
\end{aligned}
$$

$\rho_{s, i}^{p, \min } \sum_{s^{\prime} \in S_{i}^{p}} b p_{s^{\prime}, i, j, n} \leq b p_{s, i, j, n} \leq \rho_{s, i}^{p, \max } \sum_{s^{\prime} \in S_{i}^{p}} b p_{s^{\prime}, i, j, n}, \quad \forall s \in S, i \in I_{s}^{P}, j \in J_{i}, n \in N$

$$
\rho_{s, i}^{c, \min } \sum_{s^{\prime} \in S_{i}^{c}} b c_{s^{\prime}, i, j, n} \leq b c_{s, i, j, n} \leq \rho_{s, i}^{c, \max } \sum_{s^{\prime} \in S_{i}^{c}} b c_{s^{\prime}, i, j, n}, \quad \forall s \in S, i \in I_{s}^{C}, j \in J_{i}, n \in N
$$

$$
\sum_{s \in S_{i}^{p}} b p_{s, i, j, n}=\sum_{s \in S_{i}^{c}} b c_{s, i, j, n}, \quad \forall j \in J, i \in I_{j}, n \in N
$$

$$
\sum_{i \in I_{j}} b p_{s, i, j, n}=\sum_{k \in K_{j}^{p k} \cap K_{s}} K i f_{s, j, k, n}+\sum_{j^{\prime} \in J_{j}^{s e q} \cap J_{s}^{c}} J J f_{s, j, j^{\prime}, n}+U o f_{s, j, n}, \quad \forall s \in S, j \in J_{S}^{p}, n \in N
$$

$$
\sum_{i \in I_{j}} b c_{s, i, j, n}=\sum_{k \in K_{j}^{k p} \cap K_{s}} K o f_{s, k, j, n}+\sum_{j^{\prime} \in J_{j}^{s e q} \cap J_{s}^{p}} J J f_{s, j^{\prime}, j, n}+U i f_{s, j, n}, \quad \forall s \in S, j \in J_{s}^{c}, n \in N
$$

$$
s t_{s, k, n}=s t o_{s, k}+\sum_{j \in J_{k}^{p k}} \operatorname{Kif}_{s, j, k, n}+R i f_{s, k, n}-\sum_{j \in J_{k}^{k p}} K_{0} f_{s, j, k, n}-\sum_{o \in O_{s}} L f_{k, o, n}+\sum_{s^{\prime} \in S_{k}} s t d_{s^{\prime}, s, k, n}-\sum_{s^{\prime} \in S_{k}} s t d_{s, s^{\prime}, k, n}, \quad \forall s \in S, k \in K_{s}, n=1
$$

$$
s t_{s, k, n}=s t_{s, k, n-1}+\sum_{j \in J_{k}^{p k}} K i f_{s, j, k, n}+R i f_{s, k, n}-\sum_{j \in J_{k}^{k p}} K o f_{s, j, k, n}-\sum_{o \in O_{s}} L f_{k, o, n}+\sum_{s^{\prime} \in S_{k}} s t d_{s^{\prime}, s, k, n}-\sum_{s^{\prime} \in S_{k}} s t d_{s, s^{\prime}, k, n}, \quad \forall s \in S, k \in K_{s}, 1<n \leq N
$$

$$
R_{i, j}^{\min }\left(T f_{i, j, n}-T s_{i, j, n}\right) \leq \sum_{s^{\prime} \in S_{i}^{p}} b p_{s^{\prime}, i, j, n} \leq R_{i, j}^{\max }\left(T f_{i, j, n}-T s_{i, j, n}\right), \quad i \in I, j \in J_{i}, n \in N
$$

$$
\sum_{S^{\prime} \in S_{i}^{p}} b p_{s^{\prime}, i, j, n} \leq U H \times R_{i, j}^{\max } \times w v_{i, j, n}, \quad \forall i \in I, j \in J_{i}, n \in N
$$

$$
\operatorname{Kif}_{s, j, k, n} \leq V_{k}^{\max } \times i n_{s, j, k, n}, \quad \forall s \in S, j \in J_{S}^{p}, k \in K_{j}^{p k}, n \in N
$$


$\operatorname{Kof}_{s, j, k, n} \leq V_{k}^{\max } \times$ out $_{s, j, k, n}, \quad \forall s \in S, j \in J_{s}^{c}, k \in K_{j}^{k p}, n \in N$

$L f_{k, o, n} \leq V_{k}^{\max } \times l_{k, o, n}, \quad \forall k \in K_{P}, o \in O, n \in N$

$s t o_{s, k}+\sum_{j \in J_{k}^{p k}} K i f_{s, j, k, n}+R i f_{s, k, n}+\sum_{s^{\prime} \in S_{k}} s t d_{s^{\prime}, s, k, n}-\sum_{s^{\prime} \in S_{k}} s t d_{s, s^{\prime}, k, n} \leq V_{k}^{\max } y_{s, k, n}, \quad \forall s \in S, k \in K_{s}, n=1$

$s t_{s, k, n-1}+\sum_{j \in \epsilon_{k}^{p k}} K i f_{s, j, k, n}+R i f_{s, k, n}+\sum_{s^{\prime} \in S_{k}} s t d_{s^{\prime}, s, k, n}-\sum_{s^{\prime} \in S_{k}} s t d_{s, s^{\prime}, k, n} \leq V_{k}^{\max } y_{s, k, n}, \quad \forall s \in S, k \in K_{s}, 1<n \leq N$

$\sum_{j \in k_{k}^{k p}} K o f_{s, k, j, n}+\sum_{o \in O_{s}} L f_{k, o, n} \leq V_{k}^{\max } y_{s, k, n}, \quad \forall s \in S, k \in K_{s}, n \in N$

$R U_{k}^{\min }\left(\operatorname{Tof}_{k, o, n}-\operatorname{Tos}_{k, o, n}\right) \leq L f_{k, o, n} \leq R U_{k}^{\max }\left(\operatorname{Tof}_{k, o, n}-\operatorname{Tos}_{k, o, n}\right), \quad \forall k \in K_{p}, o \in O, n \in N$

$P_{s, p}^{\min } \sum_{i \in l_{s}^{p}} b p_{s, i, j, n} \leq \sum_{i \in I_{s}^{p}, s^{\prime} \in S_{i}^{c}} P_{s^{\prime}, p} b c_{s^{\prime}, i, j, n} \leq P_{s, p}^{\max } \sum_{i \in I_{s}^{p}} b p_{s, i, j, n}, \quad \forall s \in S_{A}, j \in J_{s}^{p}, n \in N$

$D_{o, s}^{-}+r_{s}-d g_{o}^{l}-r g_{s}+\leq \sum_{k, n} L f_{k, o, n}+\sum_{j, n} U o f_{s, j, n} \leq D_{o, s}^{+}+d g_{o}^{u}, \quad \forall s \in S_{A}, o \in O_{s} \cup \forall s \in S_{B}$

$\sum_{i \in I_{j}} w v_{i, j, n} \leq 1, \quad \forall j \in J, n \in N$

$\sum_{s \in K_{S}} y_{s, k, n} \leq 1, \quad \forall k \in K^{m}, n \in N$

$\sum_{s \in S_{k}} i n_{s, j, k, n}+\sum_{o \in O} l_{k, o, n} \leq 1, \quad \forall k \in K, j \in J_{k}^{p k}, n \in N$

$\sum_{k \in K_{S} \cap K_{j}^{p k}} i n_{s, j, k, n} \leq 1, \quad \forall s \in S_{A}, j \in J_{S}^{p}, n \in N$

$T f_{i, j, n} \geq T s_{i, j, n}, \quad \forall i \in I, j \in J_{i}, n \in N$

$T s_{i, j, n+1} \geq T f_{i, j, n}, \quad \forall i \in I, j \in J_{i}, n \in N, n<N$

$T s_{i, j, n+1} \geq T f_{i^{\prime}, j, n}-U H\left(1-w v_{i^{\prime}, j, n}\right), \quad \forall j \in J, i \in I_{j}, i^{\prime} \in I_{j}, i^{\prime} \neq i, n \in N, n<N$

$T f_{i, j, n}-T s_{i, j, n} \geq R L_{i} w v_{i, j, n}-U H\left(1-w v_{i, j, n}\right), \quad \forall i \in I, j \in I_{j}, n \in N$

$T s_{i, j, n} \leq T s_{i^{\prime}, j^{\prime}, n}+U H\left(2-w v_{i, j, n}-w v_{i^{\prime}, j^{\prime}, n}\right), \quad \forall j^{\prime} \in J, j \in J_{j^{\prime}}^{s e q}, i \in I_{j}, i^{\prime} \in I_{j^{\prime}}, n \in N$

$T s_{i, j, n} \geq T s_{i^{\prime}, j^{\prime}, n}-U H\left(2-w v_{i, j, n}-w v_{i^{\prime}, j^{\prime}, n}\right), \quad \forall j^{\prime} \in J, j \in J_{j^{\prime}}^{s e q}, i \in I_{j}, i^{\prime} \in I_{j^{\prime}}, n \in N$

$T f_{i, j, n} \leq T f_{i^{\prime}, j^{\prime}, n}+U H\left(2-w v_{i, j, n}-w v_{i^{\prime}, j^{\prime}, n}\right), \quad \forall j^{\prime} \in J, j \in J_{j^{\prime}}^{\text {seq }}, i \in I_{j}, i^{\prime} \in I_{j^{\prime}}, n \in N$

$T f_{i, j, n} \geq T f_{i^{\prime}, j^{\prime}, n}-U H\left(2-w v_{i, j, n}-w v_{i^{\prime}, j^{\prime}, n}\right), \quad \forall j^{\prime} \in J, j \in J_{j^{\prime}}^{\text {seq }}, i \in I_{j}, i^{\prime} \in I_{j^{\prime}}, n \in N$

$\operatorname{Tos}_{k, o, n}+U H\left(1-l_{k, o, n}\right) \geq$ times $_{o}-$ Tearly $_{o}, \quad \forall 0 \in O, k \in K_{p}, n \in N$

Tof $_{k, o, n}+U H\left(1-l_{k, o, n}\right) \leq$ timef $_{o}+$ Tlate $_{o}, \quad \forall o \in O, k \in K_{p}, n \in N$

$T s_{i, j, n} \leq U H, \quad T f_{i, j, n} \leq U H, \quad \forall i \in I, j \in J_{i}, n \in N$

$T s s_{j, k, n} \leq U H, \quad T s f_{j, k, n} \leq U H, \quad \forall j \in J, k \in K_{j}^{p k}, n \in N$

$T s s_{k, j, n} \leq U H, \quad T s f_{k, j, n} \leq U H, \quad \forall j \in J, k \in K_{j}^{k p}, n \in N$

$\operatorname{Tos}_{k, o, n} \leq U H, \quad T o f_{k, o, n} \leq U H, \quad \forall 0 \in O, \quad k \in K_{p}, \quad n \in N$

$\alpha_{j, n}, \beta_{k, n} \in \Omega_{\text {set-up }}$

$\chi_{i, i^{\prime}, j, n}, \eta_{s, s^{\prime}, k, n}, \eta o_{s, s^{\prime}, k} \in \Omega_{\text {changovers }}$

$s t d_{s, s^{\prime}, k, n} \in \Omega_{\text {giveaway }}$

$T s_{i, j, n}, T f_{i, j, n}, T s s_{j, k, n}, T s f_{j, k, n}, T s s_{k, j, n}, T s f_{k, j, n}, T o s_{k, o, n}, T o f_{k, o, n} \in \Omega_{\text {sequence }}$

The goal of the refinery operations scheduling, given in Eq. (1), is to maximize the production performance while minimizing the penalties subject to quantity, quality, and logistics giveaways and nonattainment (Kelly, 2003). The optimum values of the following variables in 
the system are driven by the shipment plan and accounts for the tradeoffs between costs of keeping inventory and changing run-modes. Constraints in the model are used to determine variables related to: (i) sequencing of tasks for units; (ii) product pool that satisfies demand orders; (iii) duration of tasks at units and duration of unloading tasks from product pools; (iv) inventory levels in component and product pools; ( $v$ ) production rates for units and unloading rate for product pools; (vi) composition of material produced and consumed.

Constraints (2a) and (2b) require that state $s$ production/consumption $b p_{s, i, j, n} / b c_{s, i, j, n}$ at unit $j$ during task $i$ and event-point $n$ be bounded by upper and lower bound on recipes and Eq. (2c) equates state produced and consumed by a unit at each event-point. Material balance constraints for unit are given by Eqs. (3a) and (3b) where $U i f_{s, j, n}$ is flow of raw material $s$ into the unit $j$ at event-point $n, k i f_{s, j, k, n}$ and $k o f_{s, k, j, n}$ represent the flow of state $s$ between unit $j$ and tank $k$ at event-point $n, J j f_{s, j, j^{\prime}, n}$ is flow of state $s$ between two consecutive units, and $U o f_{s . j, n}$ is amount of product produced that is not stored in tanks. For storage tanks, constraints (4a) and (4b) ensures material balance, where $\operatorname{Rif}_{s, k, n}$ is flow of raw material into the tank, $L f_{k, o, n}$ is product unloading from tank $k$ to satisfy demand order $o$ at event-point $n$ and $s t d_{s, s^{\prime}, k, n}$ is downgrading of the high quality product $s$ to the low quality product $s$ at event-point $n$. Total production amount is bounded by the maximum/minimum production capacity as ensured by Eq. (5), where $T s_{i, j, n} / T f_{i, j, n}$ is the start and finish time of task $i$ at unit $j$ at event-point $n$. Constrains (6) is an allocation constraint for unit and assignment constraints for flow in and out of tank is determined are given by (7a)-(7c). Here, $w v_{i, j, n}$ is a binary variable that is equal to 1 if unit $j$ is performing task $i$ at event-point $n$, binary variables $i n_{s j, k, n}$ and $o u t_{s, k, n, n}$ are equal to 1 if material is flowing in/out of tank $k$ at event point $n$ and binary $l_{k, o, n}$ is 1 if product tank $k$ fulfills demand order $o$ at event-point $n$. Constraints (8a)-(8c) determines binary assignment variable $y_{s, k, n}$ to be 1 if state $s$ is present in tank $k$ at event-point $n$. Lifting of blend product from product tank is bounded by upper and lower unloading rate, as enforced by Eq. (9) and constraint (10) ensures that blend product specification are within the bounds. Demand constraint for middle distillate products and blend products is given by Eq (11). Here, $r_{s}$ is the middle distillate product requirement, $D_{o, s}^{-} / D_{o, s}^{+}$is the lower/upper bound on the blend product for a demand order 0 . If due to production capacity limitation, demand cannot be met, positive slack variables $r g_{s}, d g_{o}^{l}$, and $d g_{o}^{u}$ are introduced to break infeasibility and obtain a feasible solution that can be implemented. Constraints (12) and (13) state that at any given event-point, only one assignment can take place. Blend product tanks are restricted from simultaneous loading and unloading of products at any given time as enforced by Eq. (14) and one flow out of blend unit restriction is imposed by constraint (16). Sequence constraints for units are given by (16a)-(16c), where they enforce finish time $T f_{i, j, n}$ of a task $i$ at unit $j$ at event-point $n$ is always greater or equal to start time $T s_{i, j, n}$ at event-point $n$ and production at next event-point $n+1$ starts after production is finished at current event-point $n$. Eq. (17) enforces minimum run-length requirement for production and blend units. For two consecutive units without any storage options in between, material flow is continuous and thus start and finish time of productions should happen at the same time at each event-point $n$ as enforced by constraints (18a)-(18d). Finished blend product demand order should to be satisfied within a fixed time window (times ${ }_{o}$ and times $_{o}$ ) as required by Eqs. (19a) and (19b). In a situation when the demand can't be met on time due to production capacity limitation, positive slack variables Tearly and Tlate $_{o}$ are introduced to obtain a feasible solution. All the timing variables are bounded by the available total scheduling horizon $U H$ as stated by Eqs. (20a)-(20d).

Additional constraints present in the full-scale model are presented in abbreviated format in Eqs. (21a)-(21d). Scheduling model includes set-up constraints for parallel units and tanks, as represented by $\Omega_{\text {set-up }}$, for multipurpose units and tanks, set of equation belonging to set $\Omega_{\text {changeovers }}$ represent changeover of service from one mode to another, and all set-ups and changeover variables are $0-1$ continuous variables. Product downgrading (giveaway) is determined using set of constraints represented by $\Omega_{\text {giveaway }}$. Sequence constraints for storage

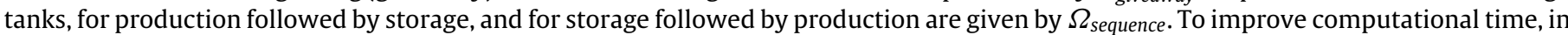
our previous work, we also proposed a set of valid inequalities to speed-up the convergence to an optimal solution. These valid inequalities are redundant in original model and not necessary however adding them improves computational time significantly. Inequalities (22a) and (22b) restate the relationship, if there is an active flow of state $s$ in or out of a tank $k$ then the state must be material present in the tank. If there is an active production task happening at unit which is producing/consuming a state that is stored in tanks at event-point $n$, then there must be at least one corresponding flow in/out of tanks at event-point $n$, this continuous flow relation is reinforced by inequalities (23a) and (23b). Product tanks can only have an active flow out of the tank if there is already product present in the tank (Eq. (24a)). There is non-simultaneous flow in and out restriction for blend product tanks, consequently demand order can only be satisfied if there was material already present the tank in previous event-point (Eq. (24b)) and before the demand order is satisfied, production should have taken place (Eq. (24c)). In our work, demand orders are expressed in chronological ascending start time of due date, hence for a particular product $s$, demand orders should be satisfied in chronological order (Eq. (24d)). For two consecutive units, production should take place simultaneously and this requirement is restated in terms of binary variable relationships in inequalities (25a)-(25d). Inequality (26) connects material consumed and produced in a unit $j$ at even-point $n$.

$$
\sum_{j \in J_{k}^{p k}} i n_{s, j, k, n} \leq \sum_{j \in J_{k}^{p k}} y_{s, k, n}, \quad \forall k \in K, s \in S_{k}, n \in N
$$

$$
\sum_{j \in J_{k}^{k p}} \text { out }_{s, j, k, n} \leq \sum_{j \in J_{k}^{k p}} y_{s, k, n}, \quad \forall k \in K, s \in S_{k}, n \in N
$$

$$
\sum_{i \in I_{j} \cap I_{s}^{p}} w v_{i, j, n} \leq \sum_{k \in K_{j}^{p k}} i n_{s, j, k, n} \leq \sum_{k \in K_{j}^{p k_{i}}} \sum_{i \in I_{j} \cap I_{s}^{p}} w v_{i, j, n}, \quad \forall j \in J, s \in S_{j}^{p}, n \in N
$$

$$
\sum_{i \in I_{j} \cap I_{s}^{c}} w v_{i, j, n} \leq \sum_{k \in K_{j}^{k p}} \text { out }_{s, k, j, n} \leq \sum_{k \in K_{j}^{k p}} \sum_{i \in I_{j} \cap I_{s}^{c}} w v_{i, j, n}, \quad \forall j \in J, s \in S_{j}^{c}, n \in N
$$

$$
\sum_{o} l_{k, o, n+1} \leq \sum_{s \in S_{k}} y_{s, k, n}, \quad \forall k \in K, n \in N, n<N
$$




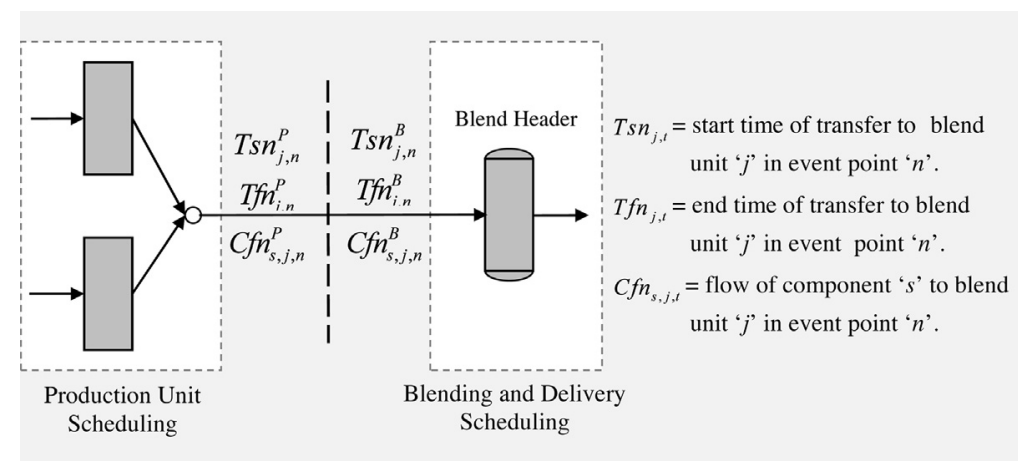

Fig. 2. Spatial decomposition of a refinery operations network.

$\sum_{o \in O_{s}} l_{k, o, n} \leq y_{s, k, n}, \quad \forall k \in K_{P}, s \in K_{s}, n \in N$

$l_{k, o, n} \leq \sum_{i \in I_{s}^{P}, j \in J_{i}, n^{\prime} \in N, n^{\prime}<n} a_{i, j, n^{\prime}}, \quad \forall s \in S_{A}, k \in K_{s}, o \in O_{s}, n \in N, n>1, \quad$ and $\quad\left[\sum_{o^{\prime} \in O_{s}} D_{o^{\prime}, s}^{-}-\sum_{k \in K_{s}} s t o_{s, k}\right]>0$

$l_{k, o^{\prime}, n} \leq \sum_{k^{\prime} \in K_{s}, n^{\prime} \in N, n^{\prime} \leq n} l_{k^{\prime}, o, n^{\prime}}, \quad \forall s \in S_{A}, k \in K_{s}, o \in O_{s}, o^{\prime} \in O_{s}, o^{\prime}>o, n \in N$

$\sum_{i \in I_{j}} w v_{i, j, n} \leq \sum_{j^{\prime} \in J^{h}, j \in j_{j^{\prime}}^{s e q}, i \in I_{j^{\prime}}} w v_{i, j^{\prime}, n}, \quad \forall j \in J^{h}, n \in N, \quad$ and $\quad \exists j^{\prime} \in J^{h}, j \in J_{j^{\prime}}^{s e q}$

$\sum_{i \in I_{j}} w v_{i, j, n} \leq \sum_{j^{\prime} \in J^{h}, j \in j_{j^{\prime}}^{s e q}, i \in I_{j^{\prime}}} w v_{i, j^{\prime}, n}, \quad \forall j \in J / J^{h}, n \in N, \quad$ and $\quad \exists j^{\prime} \in J^{h}, j \in J_{j^{\prime}}^{s e q}$

$\sum_{i \in I_{j}} w v_{i, j, n} \leq \sum_{j^{\prime} \in J^{h} \cap \int_{j}^{s e q}, i \in I_{j^{\prime}}} w v_{i, j^{\prime}, n}, \quad \forall j \in J / J^{h}, n \in N, J_{j}^{s e q} \cap J^{h} \neq \emptyset$

$\sum_{i \in I_{j}} w v_{i, j, n}=\sum_{i \in I_{j^{\prime}}} w v_{i, j^{\prime}, n}, \quad \forall j \in J / J^{h}, j^{\prime} \in J / J^{h}, j \neq j^{\prime}, j^{\prime} \in J_{j}^{s e q}, n \in N$

$\sum_{k \in K_{j}^{k p} \cap K_{s}} K o f_{s, k, j, n}+\sum_{j^{\prime} \in J_{j}^{s e q} \cap J_{s}^{p}} J J f_{s, j^{\prime}, j, n}+U i f_{s, j, n} \leq \sum_{k \in K_{j}^{p k} \cap K_{s}} K i f_{s, j, k, n}+\sum_{j^{\prime} \in J_{j}^{s e q} \cap J_{s}^{c}} J J f_{s, j, j^{\prime}, n}+U o f_{s, j, n}, \quad \forall j \in J, n \in N$

The refinery operations scheduling model (IP) includes constraints (1)-(26).

\section{Solution strategy}

The integrated refinery scheduling model (IP) gives rise to a large scale complex MILPs problem that requires specialized solution algorithms. We apply a Lagrangian decomposition (LD) algorithm to solve the integrated scheduling problem (IP) using an iterative procedure. The LD algorithm involves relaxing complicating constraints to the objective function by introducing Lagrange multipliers to form a relaxed version of a primal problem. In LD algorithm, we obtain lower bound and upper bound of the optimal value of (IP) at each iteration.

In this work, the integrated full-scale scheduling problem is decomposed into production unit scheduling problem (PSP) and blend scheduling problem (BSP) using spatial decomposition, as shown in Fig. 2. Here, the network is split into two independent sub-problems. The variables pertaining to the blend components flows amount, and start and finish times of these flows are also split alongside the connections (pipelines) in the network. Thus, two sets of linking variables, one set that belongs to the production unit scheduling subproblem (PSP) and another set that belongs to the blend scheduling sub-problem (BSP), are present. To achieve this decomposition, coupling constraints are introduced to the integrated model (IP). Coupling constraints equate the split variables (flow amount, start and end time variables) of the blend components pipelines. Based on this spatial decomposition of the refinery structure, the main goal of the production unit scheduling sub-problem is to satisfy the demand requirement of final products that belong to set $S_{B}$ and demand of the blend components required by the sub-problem. Similarly, the goal of the blend scheduling sub-problem is to satisfy the demand of the finished blend products belonging to set $S_{A}$ by mixing the raw materials, supplied by (PSP), following the blending recipe and product property specifications.

\section{Lagrangian decomposition algorithm}

Lagrangian relaxation provides an efficient way for obtaining lower bounds for large scale MILPs. These problems are characterized by a set of complicating constraints and by removing complicating constraints to the objective function using Lagrangian multipliers yield a 


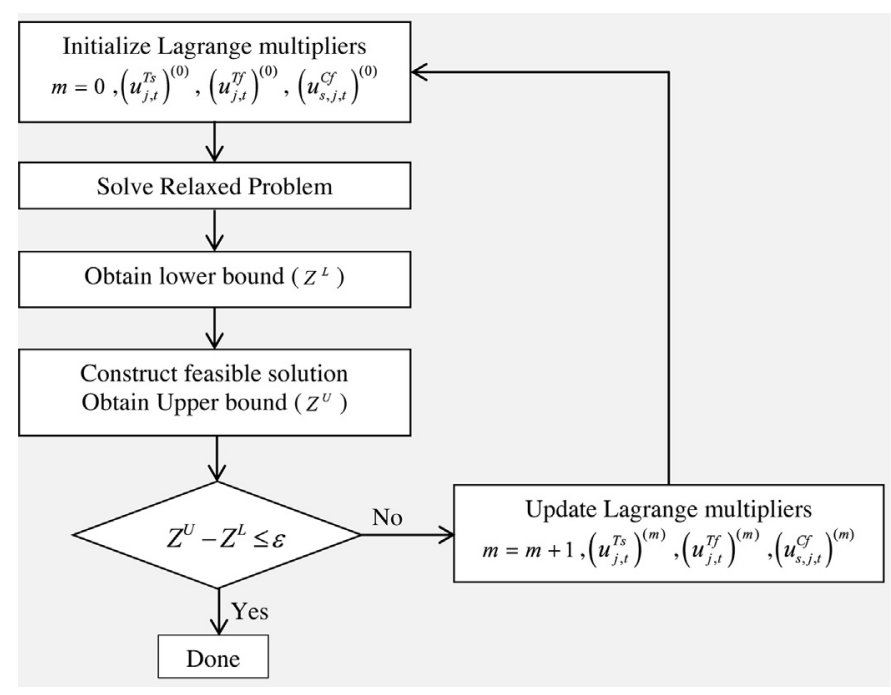

Fig. 3. Framework of classical Lagrangian decomposition algorithm.

relaxed problem that can be decomposed into smaller independent sub-problems that are easier to solve. In classical LD, lower bound is obtained from the solution of the relaxed problem and upper bound is obtained by constructing a feasible solution based on a solution to the relaxed problem. Then the multipliers are updated along a sub-gradient direction.

The general framework of the classical Lagrangian decomposition (LD) algorithm is given in Fig. 3. Here $m$ is used to account for the algorithm iterations, $\left(Z^{L}\right)^{m}$ is lower bound, and $\left(Z^{U}\right)^{m}$ is upper bound. $\left(u_{j, t}^{T s}\right)^{(m)},\left(u_{j, t}^{T f}\right)^{(m)}$, and $\left(u_{s, j, t}^{C f}\right)^{(m)}$ are the Lagrange multipliers at the $m$ th iteration.

In our work we develop a modified LD algorithm to suit the application to refinery continuous production processes, especially for refinery that have a blend header receiving at least one blend component stream directly from upstream processes without component storage. Relaxed model solution often leads to an infeasible global solution thus additional steps are needed to obtain an upper bound which is a feasible solution of the full-scale integrated model. Feasible upper bound is formulated by fixing binary variables to a solution obtained by solving the restricted relaxed sub-problems. We formulate the restricted relaxed sub-problems using the solution of Lagrangian relaxed sub-problems. The details of each step in Lagrangian algorithm are given in the following subsections.

\subsection{Relaxed problem}

The integrated full-scale model is based on continuous time representation and notion of unit specific event-points. The main goal of this section is to define the relaxed sub-problems and represent complicating variables in such a way that they are not heavily influenced by continuous time event-points at each iterations but rather actual run-modes. Before we propose a new set of time-point " $t$ " and define complicating variables using this new time-point, we layout the case for introducing this new set of time-points.

The connections between production units and blend units are cut off by splitting the pipelines between them since in our work; we focus on the refinery network that does not contain blend component tanks. As a result, the flow amount variables $\left\{J J f_{\left.s, j, j^{\prime}, n\right\}}\right\}, \forall s \in S_{b c}, j \in$ $J_{S}^{p} \cap J^{P U}, j^{\prime} \in J_{S}^{c} \cap J^{B U}, n \in N$ and the start and finish time of flow between two consecutive units (between a production unit and a blend unit) represent complicating variables. Here, $J^{B U}$ is a set of blend units and $J^{P U}$ is a set of units present in (PSP). The complicating variables are duplicated and coupling constraints are removed to objective function using Lagrangian multiplier. Resulting relaxed full-scale model is decomposed into relaxed sub-problems. However, the start and finish time between two consecutive units in the full-scale model are not expressed explicitly and to obtain a robust Lagrangian decomposition, we replaced the original complicating variables $\left\{J J f_{s, j, j^{\prime}, n}^{P}, J J f_{s, j, j^{\prime}, n}^{B}\right\}$ pertaining to the blend component flow with $\left\{C f n_{s, j^{\prime}, n}^{P}, C f n_{s, j^{\prime}, n}^{B}\right\}, \forall s \in S_{b c}, j^{\prime} \in J^{B U} \cap J_{s}^{c}, n \in N$. For the start and finish time of blend components flow between production unit operations and a blend unit, new complicating variables $\left\{T s n_{j, n}, T f n_{j, n}\right\}, \forall j \in J^{B U}, n \in N$ are introduced. These new complicating variables will be defined in the next two sub-sections in detail.

The variables pertaining to the blend component flow and start/end times of flow for all split connections between production unit operations and blend units are duplicated. The model has two sets of linking variables, one set that belongs to the production unit scheduling sub-problem $\left(C f n_{s, j, n}^{P}, T s n_{j, n}^{P}, T f n_{j, n}^{P}\right)$ and another set that belongs to the blend scheduling sub-problem $\left(C f n_{s, j, n}^{B}, T s n_{j, n}^{B}, T f n_{j, n}^{B}\right)$. All other variables in model (IP) are called non-linking variables since they are separate for both sub-problems.

Coupling constraints (27)-(29) are introduced to equate the split variables (flow amount, start and end time variables) for every event-point $n$.

$$
\begin{aligned}
& T s n_{j, n}^{P}=T s n_{j, n}^{B}, \quad \forall j \in J^{B U}, n \in N \\
& T f n_{j, n}^{P}=T f n_{j, n}^{B}, \quad \forall j \in J^{B U}, n \in N \\
& C f n_{s, j, n}^{P}=C f n_{s, j, n}^{B}, \quad \forall j \in J^{B U}, s \in S_{j}^{C}, n \in N
\end{aligned}
$$




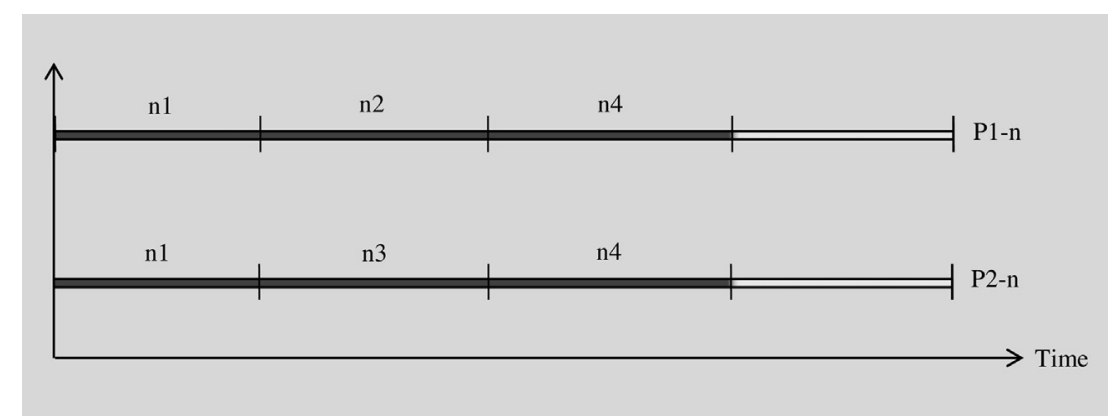

Fig. 4. Gantt chart for a blender receiving component $s$.

To obtain the Lagrangian relaxation of the original problem, the complicating constraints (27)-(29) are relaxed using Lagrange multipliers $(u)$ and considered in the objective function as shown in Eq. (30).

$$
L^{n}(u)=z+\sum_{j \in J^{B U}, n} u_{j, n}^{T s n}\left(T s n_{j, n}^{B}-T s n_{j, n}^{P}\right)+\sum_{j \in J^{B U}, n} u_{j, n}^{T f n}\left(T f n_{j, n}^{B}-T f n_{j, n}^{P}\right)+\sum_{j \in J^{B U}, s \in S_{j}^{c}, n} u_{s, j, n}^{C f n}\left(C f n_{s, j, n}^{B}-C f n_{s, j, n}^{P}\right)
$$

The objective function (30) of the relaxed problem is decomposable into smaller sub-problems corresponding to the production unit operations and the finished product blending and delivery operations, which are relatively easier to solve. The model (P1-n) includes objective function $\left(L^{B n}\right)$ and all the constraints and variables pertaining to connection in the finished product blending and delivery operations in the full-scale model (IP), whereas, the model (P2-n) includes objective function $\left(L^{P n}\right)$ and all the equations and variables pertaining to connections in the production unit operations in model (IP).

$$
\operatorname{minimize}\left\{L^{B n}(u)=z+\sum_{j \in J^{B U}, n} u_{j, n}^{T s n} T s n_{j, n}^{B}+\sum_{j \in J^{B U}, n} u_{j, n}^{T f n} T f n_{j, n}^{B}+\sum_{j \in J^{B U}, s \in S_{j}^{C}, n} u_{s, j, n}^{C f n} C f n_{s, j, n}^{B}\right\}
$$

s.t. constraints corresponding to finished product blending and delivery operations in model(IP)

$$
\operatorname{minimize}\left\{L^{P n}(u)=z-\sum_{j \in J^{B U}, n} u_{j, n}^{T s n} T s n_{j, n}^{P}-\sum_{j \in J^{B U}, n} u_{j, n}^{T f n} T f n_{j, n}^{P}-\sum_{j \in J^{B U}, s \in S_{j}^{C}, n} u_{s, j, n}^{C f n} C f n_{s, j, n}^{P}\right\}
$$

s.t. constraints corresponding to production units scheduling operations in model (IP)

The models (P1-n) and (P2-n) are independent and can be solved in parallel. Their solutions will provide a feasible solution to the original problem when the coupling constraints (27)-(29) are satisfied for all event-points. Fig. 4 shows a typical solution obtained by solving (P1-n) and (P2-n) for problem that has one blend unit, say b1. Gantt chart shows the flow amount and start/end times of one of the blend component $(s 1)$ for blend unit $b 1$. As you can see, blend components are being supplied from production units to the blend header and consumed by the blend header at the same time. However, if one were to look at Gantt chart closely, the event-points for the component streams are not the same. The complicating constraints (27)-(29) are not satisfied for event-points $n=2$ and $n=3$.

This kind of situation arises frequently when comparing solutions of (P1-n) and (P2-n) across event points due to the nature of unit specific event points and continuous time representation used in the model. To meditate this kind effect, we introduce a time-point $t$ that has one-to-one correspondence with an active event-point $n$ and cardinality of set $T$ is equal to the cardinality of set $N$. To eliminate the occurrence of non-active time-points before active time-points, additional constraints are included to enforce that active time-points always occur before non-active time-points and these constraints are presented in the next section.

To compare material flow between production operations and blend operations at each time points, a new set of variables $\left(C f_{s, j, t}, T s_{j, t}\right.$, $T f_{j, t}$ ) are introduced. The coupling constraints presented in Eq. (27)-(29) are replaced by (27b)-(29b).

$$
\begin{aligned}
& T s_{j, t}^{P}=T s_{j, t}^{B}, \quad \forall j \in J^{B U}, t \in T \\
& T f_{j, t}^{P}=T f_{j, t}^{B}, \quad \forall j \in J^{B U}, t \in T \\
& C f_{s, j, t}^{P}=C f_{s, j, t}^{B}, \quad \forall j \in J^{B U}, s \in S_{j}^{c}, t \in T
\end{aligned}
$$

The Lagrangian relaxation objective function $L^{n}(u)$ given in Eq. (30) is updated to $L(u)$ given in Eq. (30b),

$$
L(u)=z+\sum_{j \in J^{B U}, t} u_{j, t}^{T S}\left(T s_{j, t}^{B}-T s_{j, t}^{P}\right)+\sum_{j \in J^{B U}, t} u_{j, t}^{T f}\left(T f_{j, t}^{B}-T f_{j, t}^{P}\right)+\sum_{j \in J^{B U}, s \in S_{j}^{C}, t} u_{s, j, t}^{C f}\left(C f_{s, j, t}^{B}-C f_{s, j, t}^{P}\right)
$$

where the variables $\left(C f n_{s, j, n}, T s n_{j, n}, T f n_{j, n}\right)$ are replaced with variables $\left(C f_{s, j, t}, T s_{j, t}, T f_{j, t}\right)$. 
The relaxed sub-problems (P1-t) and (P2-t) are obtained by decomposing $L(u)$.

$$
\operatorname{minimize}\left\{L^{B}(u)=z+\sum_{j \in J^{B U}, t} u_{j, t}^{T s} T s_{j, t}^{B}+\sum_{j \in J^{B U}, t} u_{j, t}^{T f} T f_{j, t}^{B}+\sum_{j \in J^{B U}, s \in S_{j}^{C}, t} u_{s, j, t}^{C f} C f_{s, j, t}^{B}\right\}
$$

s.t. constraints corresponding to finished product blending and delivery operations in model

(IP) and constraints presented in Section 4.1.1

$$
\operatorname{minimize}\left\{L^{P}(u)=z-\sum_{j \in J^{B U}, t} u_{j, t}^{T s} T s_{j, t}^{P}-\sum_{j \in J^{B U}, t} u_{j, t}^{T f} T f_{j, t}^{P}-\sum_{j \in J^{B U}, s \in S_{j}^{C}, t} u_{s, j, t}^{C f} C f_{s, j, t}^{P}\right\}
$$

s.t. constraints corresponding to production units scheduling operations in model

(IP) and constraints presented in Section 4.1 .2

The Lagrangian decomposition algorithm proposed in this work is based on representing the complicating variables in terms of continuous time-point $t$.

\subsubsection{Relaxed blend scheduling problem}

Relaxed blend scheduling problem includes all the constraints and variables of the relaxed sub-problem (P1-t) and in addition to those constraints and variables, we introduce a new continuous $0-1$ variable $x_{j, n, t}^{B}$ to connect the time-points $t$ and event-points $n$.

$$
\begin{aligned}
& x_{j, n, t}^{B} \leq \sum_{i \in I_{j}} w v_{i, j, n}, \quad \forall j \in J^{B U}, n \in N, t \in T, n \geq t \\
& x_{j, n, t}^{B} \leq 1-\sum_{t^{\prime}<t, t^{\prime} \leq n} x_{j, n, t^{\prime}}^{B}, \quad \forall j \in J^{B U}, n \in N, t \in T, n \geq t \\
& x_{j, n, t}^{B} \leq 1-\sum_{n^{\prime}<n, n^{\prime} \geq t} x_{j, n^{\prime}, t}^{B}, \quad \forall j \in J^{B U}, n \in N, t \in T, n \geq t \\
& x_{j, n, t}^{B} \geq \sum_{i \in I_{j}} w v_{i, j, n}-\sum_{t^{\prime}<t, t^{\prime} \leq n} x_{j, n, t^{\prime}}^{B}-\sum_{n^{\prime}<n, n^{\prime} \geq t} x_{j, n, t^{\prime}}^{B}, \quad \forall j \in J^{B U}, n \in N, t \in T, n \geq t \\
& \sum_{n \geq t^{\prime}} x_{j, n, t^{\prime}}^{B} \leq \sum_{n \geq t} x_{j, n, t}^{B}, \quad \forall j \in J^{B U}, t \in T, t^{\prime} \in T, t^{\prime}>t \\
& x_{j, n, t}^{B}+x_{j, n^{\prime}, t^{\prime}}^{B} \leq 1, \quad \forall j \in J^{B U}, n \in N, n^{\prime} \in N, n<n^{\prime}, t \in T, t^{\prime} \in T, t^{\prime}<t, n \geq t, n^{\prime} \geq t, n^{\prime} \geq t^{\prime}, n \geq t^{\prime} \\
& x_{j, n, t}^{B}=0, \quad \forall j \in J^{B U}, n \in N, t \in T, n<t
\end{aligned}
$$

Constraint (31a) states that variable $x_{j, n, t}^{B}$ is non-zero at time-point $t$ only if a blend unit $j$ is active at event-point $n$. Here $w v_{i . j . n}$ is a binary assignment variable, 1 if task $i$ is active in unit $j$ at event-point $n$, and 0 otherwise. Constraints (31b) and (31c) enforce that each blend unit $j$ has one-to-one correspondence between a time-point and an event-point. Eq. (31d) states that if the blend unit is active at event-point $n$ then it must correspond to some time-point $t$. Constraint (31e) restrict that time-point $t^{\prime}<t$ should be assign an event-point before time-point $t$. Similar to constraint (31f), $n^{\prime}<n$ should be assigned to a time-point $t^{\prime}<t$ before assigning $n$ to $t$. Since, we want to restrict inactive time-points $\left(x_{j, n, t}^{B}=0\right)$ to happen after active time-points $\left(x_{j, n, t}^{B}=1\right)$, we add Eq. $(31 \mathrm{~g})$.

The decoupled flow and time variables at each time-point are defined as follow:

$$
\begin{aligned}
& T s_{j, t}^{B} \leq T s_{i, j, n}+U H\left(2-w v_{i, j, n}-x_{j, n, t}^{B}\right), \quad \forall j \in J^{B U}, i \in I_{j}, n \in N, t \in T, n \geq t \\
& T s_{j, t}^{B} \geq T s_{i, j, n}-U H\left(2-w v_{i, j, n}-x_{j, n, t}^{B}\right), \quad \forall j \in J^{B U}, i \in I_{j}, n \in N, t \in T, n \geq t \\
& T f_{j, t}^{B} \leq T f_{i, j, n}+U H\left(2-w v_{i, j, n}-x_{j, n, t}^{B}\right), \quad \forall j \in J^{B U}, i \in I_{j}, n \in N, t \in T, n \geq t \\
& T f_{j, t}^{B} \geq T f_{i, j, n}-U H\left(2-w v_{i, j, n}-x_{j, n, t}^{B}\right), \quad \forall j \in J^{B U}, i \in I_{j}, n \in N, t \in T, n \geq t \\
& C f_{s, j, t}^{B} \leq U i f_{s, j, n}+M\left(1-x_{j, n, t}^{B}\right), \quad \forall j \in J^{B U}, s \in S_{j}^{c}, n \in N, t \in T, n \geq t \\
& C f_{s, j, t}^{B} \geq U i f_{s, j, n}-M\left(1-x_{j, n, t}^{B}\right), \quad \forall j \in J^{B U}, s \in S_{j}^{c}, n \in N, t \in T, n \geq t \\
& T s_{j, t}^{B} \leq U H \sum_{n \geq t} x_{j, n, t}^{B}, \quad \forall j \in J^{B U}, t \in T \\
& T f_{j, t}^{B} \leq U H \sum_{n \geq t} x_{j, n, t}^{B}, \quad \forall j \in J^{B U}, t \in T
\end{aligned}
$$




$$
C f_{s, j, t}^{B} \leq M \sum_{n \geq t} x_{j, n, t}^{B}, \quad \forall j \in J^{B U}, s \in S_{j}^{c}, t \in T
$$

Constraints (32a)-(32d) assign start and end times to time-point $t$ using the corresponding event-point $n$. Similarly, blend components flow amount is assigned to time-point $t$ using Eq. (33a) and (33b), where $M$ is a big $M$ term. If a time-point is not active then the variables pertaining to blend components flows are equal to zero as defined by Eqs. (34a), (34b) and (35). If refinery under study contains multiple blend units, then the relaxed blend scheduling problem may be decomposed into multiple independent blend scheduling problems. Each of these independent blend scheduling problems must blend different components, more specifically, any two independent scheduling problems must not share a common blend component.

$$
\begin{aligned}
& \sum_{n \geq t} x_{j, n, t}^{B} \leq 1, \quad \forall j \in J^{B U}, t \in T \\
& \sum_{t \leq n} x_{j, n, t}^{B}=\sum_{i \in I_{j}} w v_{i, j, n}, \quad \forall j \in J^{B U}, n \in N
\end{aligned}
$$

To improve the computational performance of the decomposed problems, valid inequalities (36) and (37) are added to the blend scheduling models. Inequality (36) states that for a given time-point $t$, continuous $0-1$ variable $x_{j, n, t}^{B}$ can only correspond to one event-point and equality (37) states that if blend unit is active at given event-point $n$ then there must be a time-point $t$ corresponding to $n$.

\subsubsection{Relaxed production unit scheduling problem}

Continuous $0-1$ variables $x_{j, n, t}^{P}$ and $a_{j, n}$ are introduced to define split variables belonging to production unit scheduling problem in terms of time-point $t$ instead of event-point $n$. To this goal, we first introduce Eqs. (38a) and (38b).

$$
\begin{aligned}
& a_{j, n} \leq \sum_{j^{\prime} \in J^{P U}, i \in I_{j^{\prime}}, C_{j}^{c} \cap S_{i}^{p} \neq \emptyset} w v_{i, j^{\prime}, n}, \quad \forall j \in J^{B U}, n \in N \\
& \sum_{i \in I_{j^{\prime}}, S_{j}^{c} \cap S_{i^{\prime}}^{p} \neq \emptyset,} w v_{i, j^{\prime}, n} \leq a_{j, n}, \quad \forall j \in J^{B U}, j^{\prime} \in J^{P U}, j \in J_{j^{\prime}}^{s e q}, n \in N
\end{aligned}
$$

Continuous variable $a_{j, n}$ takes the value of 1 if at least one production task produces a blend component at event-point $n$ that can be consumed by blend unit $j$ as stated by constraint (38a). Eq. (38b) restricts $a_{j, n}$ to 0 if production operations are not producing blend components at event- point $n$ for blend unit $j$.

Similarly to blend scheduling problem, production unit scheduling problem has Eqs. (39a)-(39g) to define $0-1$ continuous variable $x_{j, n, t}^{P}$.

$$
\begin{aligned}
& x_{j, n, t}^{P} \leq a_{j, n}, \quad \forall j \in J^{B U}, n \in N, t \in T, n \geq t \\
& x_{j, n, t}^{P} \leq 1-\sum_{t^{\prime}<t, t^{\prime} \leq n} x_{j, n, t^{\prime}}^{P}, \quad \forall j \in J^{B U}, n \in N, t \in T, n \geq t \\
& x_{j, n, t}^{P} \leq 1-\sum_{n^{\prime}<n, n^{\prime} \geq t} x_{j, n^{\prime}, t}^{P}, \quad \forall j \in J^{B U}, n \in N, t \in T, n \geq t \\
& x_{j, n, t}^{P} \geq a_{j, n}-\sum_{t^{\prime}<t, t^{\prime} \leq n} x_{j, n, t^{\prime}}^{P}-\sum_{n^{\prime}<n, n^{\prime} \geq t} x_{j, n, t^{\prime}}^{P}, \quad \forall j \in J^{B U}, n \in N, t \in T, n \geq t \\
& \sum_{n \geq t^{\prime}} x_{j, n, t^{\prime}}^{P} \leq \sum_{n \geq t} x_{j, n, t}^{P}, \quad \forall j \in J^{B U}, t \in T, t^{\prime} \in T, t^{\prime}>t \\
& x_{j, n, t}^{P}+x_{j, n^{\prime}, t^{\prime}}^{P} \leq 1, \quad \forall j \in J^{B U}, n \in N, n^{\prime} \in N, n<n^{\prime}, t \in T, t^{\prime} \in T, t^{\prime}<t, n \geq t, n^{\prime} \geq t, n^{\prime} \geq t^{\prime}, n \geq t^{\prime} \\
& x_{j, n, t}^{P}=0, \quad \forall j \in J^{B U}, n \in N, t \in T, n<t
\end{aligned}
$$

Constraint (39a) states that variable $x_{j, n, t}^{P}$ is non-zero at time-point $t$ only if there is at least one production unit operational at event-point $n$ that can produce a blend component necessary for blend unit $j$. Constraints (39b) and (39c) enforce that there is one to one relationship between a time-point and an event-point for a every connections between production unit scheduling problem and blend unit $j$. Eq. (39d) ensures that if there is a blend component production happening event-point $n$ then it must correspond to some time-point $t$. Constraints (39e)-(39g) are same as constraints (31e)-(31g) present in the blend scheduling problem.

$$
\sum_{j^{\prime} \in J^{P U} \cap J_{s}^{p}, i \in I_{j^{\prime}} \cap l_{s}^{p}} w v_{i, j^{\prime}, n} \geq a_{j, n}, \quad \forall j \in J^{B U}, s \in S_{j}^{c}, n \in N
$$

Since there are no blend component tanks present, blend unit must receive all blend components from production scheduling problem at the same time for inline continuous blending. Constraint (32) requires that if at least one blend component is produced at event-point $n$ for a blend unit $j$ then all other blend components for the blend unit must be produced at event-point $n$. This requirement is valid because in a typical refinery, a blend unit produces multiple products by mixing a fixed set of blend components using different blend recipes. That is, $\forall j \in J^{B U}, I \in I_{j}$, the blend components that can be consumed by different tasks at blend unit $j$ gives us $S_{j}^{c}=S_{i}^{c}$. 
The goals of the production unit scheduling problem is to produce hydrocarbon products $\left(S_{B}\right)$ that are not stored in tanks but are supplied straight to the market and to produce blend components that are needed by blend units to produce finished blend products. Since there are no tanks available to store blend components, these products (blend components) are supplied straight to the market (here market is blend unit). Thus, the product set $\left(S_{B}\right)$ includes the blend components and other hydrocarbon products that will be used as feedstock in chemical industry. Constraints relating the split variables (flow, start and end times) of the production scheduling problem to time-points are given below.

$$
\begin{aligned}
& T s_{j, t}^{P} \leq U H \sum_{n \geq t} x_{j, n, t}^{P}, \quad \forall j \in J^{B U}, t \in T \\
& T f_{j, t}^{P} \leq U H \sum_{n \geq t} x_{j, n, t}^{P}, \quad \forall j \in J^{B U}, t \in T \\
& T s_{j^{\prime}, t}^{P} \leq T s_{i, j, n}+U H\left(2-w v_{i, j, n}-x_{j^{\prime}, n, t}^{P}\right), \quad \forall j \in J^{P U}, j^{\prime} \in J^{B U}, j^{\prime} \in J_{j}^{s e q}, i \in I_{j}, n \in N, t \in T, n \geq t, S_{i}^{p} \cap S_{j^{\prime}}^{c} \neq \emptyset \\
& T s_{j^{\prime}, t}^{P} \geq T s_{i, j, n}-U H\left(2-w v_{i, j, n}-x_{j^{\prime}, n, t}^{P}\right), \quad \forall j \in J^{P U}, j^{\prime} \in J^{B U}, j^{\prime} \in J_{j}^{s e q}, i \in I_{j}, n \in N, t \in T, n \geq t, S_{i}^{p} \cap S_{j^{\prime}}^{c} \neq \emptyset \\
& T f_{j^{\prime}, t}^{P} \leq T f_{i, j, n}+U H\left(2-w v_{i, j, n}-x_{j^{\prime}, n, t}^{P}\right), \quad \forall j \in J^{P U}, j^{\prime} \in J^{B U}, j^{\prime} \in J_{j}^{s e q}, i \in I_{j}, n \in N, t \in T, n \geq t, S_{i}^{p} \cap S_{j^{\prime}}^{c} \neq \emptyset \\
& T f_{j^{\prime}, t}^{P} \geq T f_{i, j, n}-U H\left(2-w v_{i, j, n}-x_{j^{\prime}, n, t}^{P}\right), \quad \forall j \in J^{P U}, j^{\prime} \in J^{B U}, j^{\prime} \in J_{j}^{s e q}, i \in I_{j}, n \in N, t \in T, n \geq t, \quad S_{i}^{p} \cap S_{j^{\prime}}^{c} \neq \emptyset
\end{aligned}
$$

Constraints (33a) and (33b) states that start and end times are zero if material is not flowing to a blend unit $j^{\prime}$ at time-point $t$. If production unit operations are producing blend components at time-point $t$, then Eqs. (34a)-(34d) are used to assign start and end times to blend component flow to a blend unit $j^{\prime}$.

All production and blend units in the refinery are continuous processes and since there is no storage between the production unit scheduling operations and the blend units, material produced by the production unit scheduling problem is directly consumed. Thus production capacity of blend units and blend recipes of each blend component should also be taken into consideration when the components are supplied by production unit operations. Parameters $R B_{j}^{\max }$ and $R B_{j}^{\min }$ are maximum and minimum blend rate of a blend unit $j$, respectively and are calculated using Eqs. (35a) and (35b). The maximum and minimum blend recipe of component $s$ in blend unit $j, \rho b_{s, j}^{\max }$ and $\rho b_{s, j}^{\min }$ respectively, are determined from Eqs. (36a) and (36b).

$$
\begin{aligned}
& R B_{j}^{\max }=\max _{i \in I_{j}}\left(R_{i, j}^{\max }\right), \quad \forall j \in J^{B U} \\
& R B_{j}^{\min }=\min _{i \in I_{j}}\left(R_{i, j}^{\min }\right), \quad \forall j \in J^{B U} \\
& \rho b_{s, j}^{\max }=\max _{i \in I_{j}}\left(\rho_{s, i}^{\max }\right), \quad \forall j \in J^{B U} \\
& \rho b_{s, j}^{\min }=\min _{i \in I_{j}}\left(\rho_{s, i}^{\min }\right), \quad \forall j \in J^{B U}
\end{aligned}
$$

The blend component production is set to zero by Eq. (37a) if there is no production happening at time-point $t$ for blend component $s$. Constraints (37b) and (37c) determine the individual component flow amount from the production unit scheduling problem to a blend unit $j$ at time-point $t$.

$$
\begin{aligned}
& C f_{s, j, t}^{P} \leq \rho b_{s, j}^{\max } R B_{j}^{\max } U H \sum_{n \geq t} x_{j, n, t}^{P}, \quad \forall j \in J^{B U}, s \in S_{j}^{c}, t \in T \\
& C f_{s, j, t}^{P} \leq \sum_{j^{\prime} \in J_{s}^{p}} U o f_{s, j^{\prime}, n}+\rho b_{s, j}^{\max } R B_{j}^{\max } U H\left(1-x_{j, n, t}^{P}\right), \quad \forall j \in J^{B U}, s \in S_{j}^{c}, n \in N, t \in T, n \geq t \\
& C f_{s, j, t}^{P} \geq \sum_{j^{\prime} \in j_{s}^{p}} U o f_{s, j^{\prime}, n}-\rho b_{s, j}^{\max } R B_{j}^{\max } U H\left(1-x_{j, n, t}^{P}\right), \quad \forall j \in J^{B U}, s \in S_{j}^{c}, n \in N, t \in T, n \geq t
\end{aligned}
$$

$$
\sum_{s \in S_{j}^{c}} C f_{s, j, t}^{P} \leq R B_{j}^{\max }\left(T f_{j, t}^{P}-T s_{j, t}^{P}\right)+R B_{j}^{\max } U H\left(1-\sum_{n \geq t} x_{j, n, t}^{P}\right), \quad \forall j \in J^{B U}, t \in T
$$

$$
\sum_{s \in S_{j}^{c}} C f_{s, j, t}^{P} \geq R B_{j}^{\min }\left(T f_{j, t}^{P}-T s_{j, t}^{P}\right)-R B_{j}^{\min } U H\left(1-\sum_{n \geq t} x_{j, n, t}^{P}\right), \quad \forall j \in J^{B U}, t \in T
$$

$$
C f_{s, j, t}^{P} \leq \rho b_{s, j}^{\max } R B_{j}^{\max }\left(T f_{j, t}^{P}-T s_{j, t}^{P}\right)+\rho b_{s, j}^{\max } R B_{j}^{\max } U H\left(1-\sum_{n \geq t} x_{j, n, t}^{P}\right), \quad \forall j \in J^{B U}, s \in S_{j}^{c}, t \in T
$$

$$
C f_{s, j, t}^{P} \geq \rho b_{s, j}^{\min } R B_{j}^{\min }\left(T f_{j, t}^{P}-T s_{j, t}^{P}\right)-\rho b_{s, j}^{\min } R B_{j}^{\min } U H\left(1-\sum_{n \geq t} x_{j, n, t}^{P}\right), \quad \forall j \in J^{B U}, s \in S_{j}^{c}, t \in T
$$


Constraints (38a) and (38b) enforce that total amount of material supplied to a blend unit $j$ at time-point $t$ is bounded by maximum and minimum production rate of corresponding blend unit and production of each blend component is bounded by Eqs. (38c) and (38d).

Similar to valid inequalities (36) and (37) included in blend scheduling sub-problem, we introduce constraints (39) and (40) to production unit scheduling problem. Inequality (39) states that for a given time-point $t$, continuous $0-1$ variable $x_{j, n, t}^{P}$ can only correspond to one eventpoint. If at event-point $n$ production unit operations are producing materials for blend unit $j$, then there must be at least one time-point $t$ corresponding to $n$ as stated by equality (40).

$$
\begin{aligned}
& \sum_{n \geq t} x_{j, n, t}^{P} \leq 1, \quad \forall j \in J^{B U}, t \in T \\
& \sum_{t \leq n} x_{j, n, t}^{P}=a_{j, n}, \quad \forall j \in J^{B U}, n \in N
\end{aligned}
$$

\subsection{Construction of a feasible schedule}

Traditionally an upper bound for the Lagrangian decomposition is obtained by fixing certain binary variables of the original integrated MILP problem (IP) based upon the solution of the relaxed sub-problems. However the solution derived by solving relaxed sub-problems $((\mathrm{P} 1-\mathrm{t})$ and (P2-t)) are usually infeasible for global problem in each iteration because the complicating constraints are violated. Therefore, a heuristics procedure or some other method for generating a feasible solution is necessary. In this work, a feasible solution at each iteration is obtained by fixing some of the binary variables to 1 and to decide which binaries to fix, we propose using solution of the restricted relaxed sub-problems. These restricted relaxed sub-problems' solution will always provide a feasible solution to the full-scale model unlike the relaxed sub-problems. The restricted relaxed sub-problems are formulated by fixing some of the binary and $0-1$ continuous variables in the relaxed sub-problems.

In proposed decomposition strategy, two relaxed sub-problems are solved independent of each other, thus the schedule obtained solving the relaxed problems (P1-t) and (P2-t) may lead to an infeasible solution of (IP). In most instances the infeasibility in the full-scale model occurs because the material balance constraints are not satisfied for blend units or production units that produce blend components. The refinery system under study does not contain blend component tanks therefore blend operations and corresponding production unit operations must operate concurrently to satisfy mass balance constraints of rundown streams. The relaxed sub-problems (P1-t) and (P2-t) defined in previous section are solved independent of each other, thus at some iteration $m$, below scenario may happen.

The production operations scheduling model (P2-t) produces a solution where blend components are supplied to a blend unit $j$ during event-points $n=2$ and $n=4$, that is $x_{j, 2,1}^{P}=1$ and $x_{j, 4,2}^{P}=1$, while the relaxed blend scheduling model (P1-t) solution has the blend unit $j$ is receiving components during event-points $n=1, n=3$, and $n=4$ that is $x_{j, 1,1}^{B}=1, x_{j, 3,2}^{B}=1$, and $x_{j, 4,3}^{B}=1$.

In above scenario, if the binary variables $\left\{w v_{i, j, n}\right\} \quad \forall i, j, n$ in the original integrated model (IP) are fixed based upon solutions of the relaxed sub-problems, it would lead to an infeasible global solution. Infeasibility occurs because in the full-scale model, for two sequential units without storage any between them must operate at the same event-point. In this scenario, the blend unit is consuming material at event-points $n 1, n 3$, and $n 4$ while the blend unit is only receiving components at event-points $n 2$ and $n 4$ from production unit operations. Hence to obtain a global feasible full-scale solution, production unit operations must supply blend components at the same event-point as when they are being consumed by a blend unit.

In this work, to build a feasible global solution and to obtain an upper bound, instead of using all heuristics rules, we fixed certain variables in one of the relaxed sub-problems and solve this restricted-relaxed sub-problem to optimality. Using this approach negates the need to develop some heuristics criteria to obtain upper bound (global feasible solution) in LD algorithm.

In the scenario described above, material balance equation for a blend unit and corresponding consecutive production units that produce blend components would not be satisfied at event-points $n 1, n 2$, and $n 3$. Therefore, when we solve the restricted relaxed production unit scheduling problem to optimality, the resulting new solution must have production unit operations supplying blend components to a blend-unit at event-points $n 1, n 3$, and $n 4$. Based on these new results of restricted relaxed sub-problem (P2-t) and original relaxed subproblem (P1-t) results, if we fixed binary variables at event-points $n 1, n 3$, and $n 4$ in the full-scale model (IP), it would provide a feasible solution for the scenario presented earlier.

Before the steps to build restricted relaxed sub-problems are presented, we first state assumptions behind the proposed method to derive restricted relaxed sub-problems.

We assume that there are no parallel blend units present in the refinery and no two blend units share a particular blend component. The mathematical representation of these assumptions is given below as:

$$
S_{j}^{c} \cap S_{j^{\prime}}^{c}=\emptyset, \quad K_{j}^{p k} \cap K_{j^{\prime}}^{p k}=\emptyset, \quad \forall j \in J^{B U}, j^{\prime} \in J^{B U}, j \neq j^{\prime}
$$

These assumptions allow us to decompose blend scheduling problems (BSP) into sub-problems containing only one blend unit. Here each (BSP) sub-problem defined as $B S P^{j}$ has its own set of blend components, materials, and tanks corresponding to a blend unit $j \in J^{B C}$. Thus, the blend scheduling problem (BSP) can be decomposed into total of $U^{B C} \mid$ sub-problems; here $J^{B C}$ is a set of blend units.

Now we analyze the structure of the (PSP), particular for a refinery that has more than one blend unit and the (BSP) can be decomposed into independent sub-problems. We check if the production units that supply components to different (BSP) sub-problems are connected or not. Production units are said to be "connected" if they all have to be operational at the same time which corresponds to being active at same event-points so that the continuous process operational rule is not violated for the (PSP). We assume in our work that all the production units which produce blend components for different blend units are connected. Based upon this assumption, all independent sub-problems of the (BSP) must also be active at the same event-points to obtain a feasible solution to the integrated model (IP).

A two steps approach is developed to determine an upper bound for the algorithm which is obtained from a feasible solution to the original full-scale model. In step 1 a restricted relaxed problem is solved to provide a solution that has production units active at the same event-points as the blend units. Using the solution of step 1, in step 2 a feasible solution to (IP) is realized. 


\subsubsection{Restricted relaxed problem}

Upon solving relaxed sub-problems presented in Section 4.1, we obtain schedule for blending operations and production unit operations, respectively. To build a restricted relaxed problem using the optimal solution of relaxed problem, the following steps are proposed:

Step 1: From the optimal relaxed sub-problems solution, determine over the scheduling horizon which tasks, units, and storage tanks are active for at least one event-point.

Step 2: Pick a primary sub-problem from $\{P S P\} \cup\left\{B S P^{j}, \forall j \in J^{B U}\right\}$ by analyzing solution of the corresponding relaxed sub-problems. Determine active event-points during which the primary sub-problem is either supplying or receiving blend components.

Step 3: Obtain restricted relaxed problem by fixing some of the binary variables in corresponding relaxed problem to 1 at active eventpoints.

Each step is described in detail as follow:

Step 1: Determine parameters from an optimal solution of relaxed sub-problems. using Eqs. (41a)-(41d), we can calculate active task for units $\left(w v_{i, j}^{a}\right)$, the flow in and out of tanks $\left(i n_{s, j, k}^{a}\right.$ and out $\left.t_{s, k, j}^{a}\right)$, and the changeovers from task $i$ to $i^{\prime}$ at unit $j$, respectively over the scheduling horizon. Parameter $\bar{\chi}_{i, i^{\prime}, j}^{a}$ is calculated using Eq. (41e) which gives the total number of time a task $i$ is change to a task $i^{\prime}$ at unit $j$.

$$
\begin{aligned}
& w v_{i, j}^{a}=\left\{\begin{array}{l}
1 \text { if } \sum_{n} w v_{i, j, n}>0 \\
0 \text { if } \sum_{n} w v_{i, j, n}=0
\end{array}, \quad \forall j \in J, i \in I_{j}\right. \\
& i n_{s, j, k}^{a}=\left\{\begin{array}{l}
1 \text { if } \sum_{n} i n_{s, j, k . n}>0 \\
0 \text { if } \sum_{n}^{n} i n_{s, j, k, n}=0
\end{array}, \quad \forall s \in S, k \in K_{s}, j \in J_{k}^{p k}\right. \\
& o u t_{s, k, j}^{a}=\left\{\begin{array}{ll}
1 & \text { if } \sum_{n} \text { out }_{s, k, j, n}>0 \\
0 & \text { if } \sum_{n} \text { out }_{s, k, j, n}=0
\end{array} \quad \forall s \in S, k \in K_{s}, j \in J_{k}^{k p}\right. \\
& \chi_{i, i^{\prime}, j}^{a}=\left\{\begin{array}{l}
1 \text { if } \sum_{n} \chi_{i, i^{\prime}, j, n}>0 \\
0 \text { if } \sum_{n} \chi_{i, i^{\prime}, j, n}=0
\end{array}, \quad \forall j \in J, i \in I_{j}, i^{\prime} \in I_{j}\right. \\
& \bar{\chi}_{i, i^{\prime}, j}^{a}=\sum_{n} \chi_{i, i^{\prime}, j, n}, \quad \forall j \in J, i \in I_{j}, i^{\prime} \in I_{j}
\end{aligned}
$$

Step 2: From the solution of relaxed sub-problems, the total number of active time-points for each Lagrangian decomposed problem is calculated as:

$$
\text { Tat }_{j}=\sum_{t, n \geq t} x_{j, n, t}, \quad \forall j \in J^{B U}
$$

Since we assume that the production units which produce blend components for different blend units are connected, for (PSP),

$$
\operatorname{Tat}^{P}=\operatorname{Tat}_{j}^{P}=\operatorname{Tat}_{j^{\prime}}^{P}, \quad \forall j \in J^{B U}, j^{\prime} \in J^{B U}, j \neq j^{\prime}
$$

The total numbers of active-points for each sub-problem $B S P^{j}$ containing blend unit $j$ are given as Tat ${ }_{j}^{B}$. The maximum number of active time-points is determined by finding maximum among (BSP) and (PSP) as:

$$
\text { Mat }=\max \left[\left\{\operatorname{Tat}^{P}\right\} \cup\left\{\operatorname{Tat}_{j}^{B}, \forall j \in J^{B U}\right\}\right]
$$

If there is more than one sub-problem that has the total active time-points equal to Mat, then the following rules are used to determine the primary problem:

Rule 1: If Tat ${ }_{j}^{B}=$ Mat and sub-problem $B S P^{j}$ includes multipurpose blend unit $j$ then the primary problem is $\left(P P=\left\{B S P^{j}\right\}\right)$.

Rule 2: If $\mathrm{Tat}_{j}^{B}=$ Mat and sub-problem $B S P^{j}$ does not include multipurpose blend unit $j$ then the blend scheduling sub-problem is chosen as the primary problem $\left(P P=\left\{B S P^{j}\right\}\right)$.

Rule 3: If Tat $_{j}^{B} \neq$ Mat and Tat ${ }^{P}=$ Mat then (PSP) is selected as the primary problem $(P P=\{P S P\})$.

When determining the primary problem, priority is given to a problem that satisfy rule 1 ; however if rule 1 is not satisfy then we check rule 2 and subsequently rule 3 . 
The solution of primary problem is used to determine parameter $A e_{n}$ which represents active event-point.

$$
\begin{aligned}
& A e_{n}=\left\{\begin{array}{ll}
1 & \text { if } \sum_{i \in I_{j}} w v_{i, j, n}>0 \\
0 & \text { if } \sum_{i \in I_{j}} w v_{i, j, n}=0
\end{array}, \quad \forall j \in J^{B C}, B S P^{j} \in P P\right. \\
& A e_{n}=\left\{\begin{array}{l}
1 \text { if } \sum_{j^{\prime} \in J^{P U}, j \in \epsilon_{j^{\prime}}^{s e q}, i \in I_{j^{\prime}}, S_{j}^{c} \cap S_{i}^{p} \neq \emptyset} w v_{i, j^{\prime}, n}>0 \\
0 \quad \text { if } \sum_{j^{\prime} \in J^{P U}, j \in \epsilon_{j^{\prime}}^{s e q}, i \in I_{j^{\prime}}, S_{j}^{c} \cap S_{i}^{p} \neq \emptyset} w v_{i, j^{\prime}, n}=0
\end{array}, \quad \forall j \in J^{B C}, P S P \in P P\right.
\end{aligned}
$$

Parameter $A e_{n}$ is determined by Eq. (42a) if the primary problem is a sub-problem $B S P^{j}$ otherwise it is determined by Eq. (42b).

Step 3: Using parameters $w v_{i, j}^{a}, i n_{s, j, k}^{a}$, out $t_{s, k, j}^{a}, \chi_{i, i^{\prime}, j}^{a}, \bar{\chi}_{i, i^{\prime}, j}^{a}$ and $A e_{n}$ we restrict the solution space of the relaxed sub-problems so that a solution of the restricted problem have production operations producing blend components at the same event-point as the blend units are mixing. We fix binary variables $\left\{w v_{i, j, n}, i_{s, j, k, n}, o u t_{s, k, j, n}, \chi_{i, i^{\prime}, j, n}\right\}$ as shown in Eqs. (43a)-(43h).

$$
\begin{aligned}
& w v_{i, j, n}=w v_{i, j}^{a} A e_{n}, \quad \forall j \in J, j \notin J^{m}, i \in I_{j}, n \in N \\
& w v_{i, j, n}=0, \quad \forall j \in J^{m}, i \in I_{j}, n \in N,\left(A e_{n}=0\right) \vee\left(w v_{i, j}^{a}=0\right) \\
& \sum_{i \in J_{j}, w v_{i, j}^{a}>0} w v_{i, j, n}=A e_{n}, \quad \forall j \in J^{m}, n \in N, A e_{n}>0 \\
& \sum_{n, A e_{n}>0} w v_{i, j, n} \geq w v_{i, j}^{a}, \quad \forall j \in J^{m}, i \in I_{j}, w v_{i, j}^{a}>0, \sum_{i^{\prime}, i^{\prime \prime}} \bar{\chi}_{i^{\prime}, i^{\prime \prime}, j}=0 \\
& \chi_{i, i^{\prime}, j, n}=0, \quad \forall j \in J, i \in I_{j}, i^{\prime} \in I_{j}, n \in N,\left(A e_{n}=0\right) \vee\left(\chi_{i, i^{\prime}, j}^{a}=0\right) \\
& \sum_{n, A e_{n}>0} \chi_{i, i^{\prime}, j, n}=\bar{\chi}_{i, i^{\prime}, j}^{a}, \quad \forall j \in J, i \in I_{j}, i^{\prime} \in I_{j}, i \neq i^{\prime}, n \in N, \chi_{i, i^{\prime}, j}^{a}>0 \\
& i n_{s, j, k, n}=i n_{s, j, k}^{a} A e_{n}, \quad \forall k \in K, k \notin K^{m}, k \notin K_{p}, j \in J_{k}^{p k}, j \notin J^{m}, i \in I_{j}, s \in S_{k}, n \in N \\
& \text { out }_{s, k, k, n}=\text { out }_{s, k, j}^{a} A e_{n}, \quad \forall k \in K, k \notin K^{m}, k \notin K_{p}, j \in J_{k}^{k p}, j \notin J^{m}, i \in I_{j}, s \in S_{k}, n \in N
\end{aligned}
$$

Eq. (43a) fixes binary assignment variable for all units except for multipurpose units. For multipurpose units, Eqs. (43b)-(43f) are used, where Eq. (43b) fixes assignment variable $w v_{i, j, n}$ to zero if the task $i$ has not been active in relaxed problem or if the event-point $n$ is not active in primary problem. Constraint (43c) forces unit to be active when $A e_{n}=1$ and constraint (43d) enforces that task $i$ needs be active in unit $j$ if $w v_{i, j}^{a}=1$ and no tasks changeovers are happening $\sum_{i^{\prime}, i^{\prime \prime}} \bar{\chi}_{i^{\prime}, i^{\prime \prime}, j}^{a}=0$ over the time horizon. Eq. (43e) fixes continuous $0-1$ variable $\chi_{i, i^{\prime}, j, n}$ to be zero at event-point $n$ if $A e_{n}=0$ and the total number times the changeover from task $i$ to $i^{\prime}$ can happen in unit $j$ is fixed to $\bar{\chi}_{i, i^{\prime}, j}^{a}$ in constraint (43f). Binary variables $i n_{s, j, k, n}$ and $o u t_{s, k, j, n}$ are fixed in restricted relaxed problem as shown in Eqs. (43g)-(43h) for all units and tanks that do not perform multipurpose tasks and store final product tanks. Since the blend products demand is to be satisfied before its due date, we do not fix any variables associated with the product tanks as not to restrict solution space of the restricted relaxed problem too much that product demands do not get satisfied. This situation can occur because in refinery under study, the products tanks cannot load and unload products simultaneously. Thus, if we fix the binary variable associated with loading and unloading of product tanks based upon the solution of the relaxed problem, it may cause infeasibility in the restricted relaxed problem.

Restricted relaxed problem is built and solved for every sub-problem except for the sub-problem that is chosen as the primary problem. Solution of the restricted relaxed problem gives us consistent solution across (BSP) and (PSP) in terms of event-points so that the continuous process operational rule for two consecutive units is not violated.

\subsubsection{Upper bounding problem}

We fix many of the binary variables $\left\{w v_{i, j, n}, i n_{s, j, k, n}, o u t_{s, k, j, n}, l_{k, o, n}\right\}$ in problem (IP) to the values of the corresponding binary variables obtained from the solution of restricted relaxed sub-problems and obtain a MILP model (IP-U). The solution of the restricted relaxed problem is $\left\{w v_{i, j, n}^{r}, i n_{s, j, k, n}^{r}\right.$, out $\left.t_{s, k, j, n}^{r}, l_{k, o, n}^{r}\right\}$ and based on non-zero values of these binaries, corresponding variables in (IP) are fixed. Only certain binary variables in problem (IP) are fixed to 1 and no binaries are fixed to 0 so that a better upper bound can be attained in earliest iterations in the LD algorithm. Eqs. (44a)-(44d) show how the variables are fixed in the problem (IP) to obtain a MILP model (IP-U).

$$
\begin{aligned}
& w_{i, j, n}=1, \quad \forall j \in J, i \in I_{j}, n \in N, w v_{i, j, n}^{r}>0 \\
& i n_{s, j, k, n}=1, \quad \forall k \in K, j \in J_{k}^{p k}, i \in I_{j}, s \in S_{k}, n \in N, i_{s, j, k}^{r}>0 \\
& \text { out }_{s, k, k, n}=1, \quad \forall k \in K, j \in J_{k}^{k p}, i \in I_{j}, s \in S_{k}, n \in N, \text { out }_{s, k, j}^{r}>0 \\
& l_{k, o, n}=1, \quad \forall k \in K_{p}, j \in J_{k}^{p k}, i \in I_{j}, s \in S_{k}, n \in N, l_{k, o, n}^{r}>0
\end{aligned}
$$


Solving model (IP-U) yields an upper bound on the solution of (IP). The model (IP-U) is always feasible because the model includes demand, due-dates, and blend logistics giveaways.

\subsection{Strengthening the Lagrangian decomposition}

There are several constraints, redundant or unnecessary in (IP) that can be added to the relaxed problem and the restricted relaxed problem to strengthen the performance of the Lagrangian algorithm. To improve the lower bound, we propose a pre-processing step before the Lagrangian algorithm and add constraints related to product demands to the decomposed problems in the algorithm. A common feature of the preprocessing steps and constraints strengthening lower bound is that they in some sense recreate what has been relaxed, namely the network structure of the refinery, but without destroying the separability of the Lagrangian sub-problems. Furthermore, using the solution of the best upper bound, in the decomposed problems, penalty variables relating violations of demands, due dates, and blend logistics can be fixed to zero.

\subsubsection{Preprocessing step}

Because the refinery is decomposed into (BSP) and (PSP), production capacity limitation for the blend components in (PSP) is not taken into consideration while solving (BSP). Therefore, we present a preprocessing step that determines the maximum production rate for each blend component $s$ and maximum production capacity for a set of blend components that can be mixed by a blend unit.

$$
\begin{aligned}
& \operatorname{minimize}\left\{z_{s}^{P P}=-100 \sum_{j \in J^{P U}, j \in J_{s}^{p}, n \in N} U o f_{s, j, n}\right\}, \quad \forall s \in S_{b c} \\
& \operatorname{minimize}\left\{z_{j}^{P P}=-100 \sum_{j^{\prime} \in J^{P U}, s \in S_{j}^{C} \cap S_{j^{\prime}}^{p}, n \in N} U o f_{s, j, n}\right\}, \quad \forall j \in J^{B U}
\end{aligned}
$$

The preprocessing problem (PC-s) and (PC-j) are based upon the relaxed sub-problem corresponding to production unit operations (PSP). The model (PC-s) is obtained by replacing objective function of relaxed problem with that given in Eq. (45) to determine maximum production rate of each blend component. Another preprocessing problem (PC-j) is presented to calculate the maximum rate at which production units can supply materials to each blend unit. The objective function for (PC-j) is presented in Eq. (46). Problem (PC-s) is solved for each blend component and problem (PC-j) for each blend unit before the Lagrangian algorithm begins. Furthermore these problems are independent of each other and can be solved in parallel.

Upon solving the pre-processing problems, we obtain values of variables $\left\{C f_{s, j, t}^{P}, T s_{j, t}^{P}, T f_{j, t}^{P}, x_{j, n, t}^{P}\right\}, \quad \forall s, j, n, t$ from the optimal solution.

$$
\begin{aligned}
& R P_{s}^{\max }=\max _{t}\left\{\frac{C f_{s, j, t}^{P} \sum_{n} x_{j, n, t}^{P}}{T f_{j, t}^{P}-T s_{j, t}^{P}}\right\}, \quad \forall j \in J^{B U}, s \in S_{j}^{c} \\
& R P_{j}^{\max }=\max _{t}\left\{\frac{\sum_{s \in S_{j}^{c}} C f_{s, j, t}^{P} \sum_{n} x_{j, n, t}^{P}}{T f_{j, t}^{P}-T s_{j, t}^{P}}\right\}, \quad \forall j \in J^{B U}
\end{aligned}
$$

The maximum rate of production of component sis determined by Eq. (47a) using the optimal solution obtained upon solving corresponding preprocessing problem (PC-s). Similarly, the maximum rate for set of blend components supplied to blend unit $j$ is determined by Eq. (47b) using the optimal solution values of corresponding preprocessing problem (PC-j).

\subsubsection{Strengthening lower bound}

To strengthen the Lagrangian decomposition algorithm, the information obtained in pre-processing step is applied to update the blend component production rate upper bounds in Eqs. (33a), (33b), and (35) for relaxed and restricted relaxed blend scheduling problems. Computationally inefficient big $M$ term is replaced with tighter bound upper $R P_{s}^{\max }$ as shown in Eqs. (48a)-(48c).

$$
\begin{aligned}
& C f_{s, j, t}^{B} \leq U i f_{s, j, n}+R P_{s}^{\max }\left(1-x_{j, n, t}^{B}\right), \quad \forall j \in J^{B U}, s \in S_{j}^{c}, n \in N, t \in T, n \geq t \\
& C f_{s, j, t}^{B} \geq U i f_{s, j, n}-R P_{s}^{\max }\left(1-x_{j, n, t}^{B}\right), \quad \forall j \in J^{B U}, s \in S_{j}^{c}, n \in N, t \in T, n \geq t \\
& C f_{s, j, t}^{B} \leq R P_{s}^{\max } \sum_{n \geq t} x_{j, n, t}^{B}, \quad \forall j \in J^{B U}, s \in S_{j}^{c}, t \in T \\
& C f_{s, j, t}^{B} \leq R P_{s}^{\max }\left(T f_{j, t}^{B}-T s_{j, t}^{B}\right)+R P_{s}^{\max } U H\left(1-\sum_{n \geq t} x_{j, n, t}^{B}\right), \quad \forall j \in J^{B U}, s \in S_{j}^{c}, t \in T \\
& \sum_{s \in S_{j}^{c}} C f_{s, j, t}^{B} \leq R P_{j}^{\max }\left(T f_{j, t}^{B}-T s_{j, t}^{B}\right)+R P_{j}^{\max } U H\left(1-\sum_{n \geq t} x_{j, n, t}^{B}\right), \quad \forall j \in J^{B U}, t \in T
\end{aligned}
$$


The amount of blend component processed by blend unit $j$ at time-point $t$ is bounded by the maximum production rate of the component and blend unit's process time as enforced by constraint (48d). Similarly, constraint (48e) states that the total bending rate is bounded by the maximum rate at which material is supplied to a blend unit $j$.

$$
\begin{aligned}
& \sum_{i \in I_{s}^{p}, j \in J_{i}, n} w v_{i, j, n} \geq 1, \quad \forall s \in S_{A} \cup S_{B}, A m t^{\min }>0 \\
& \sum_{i \in \epsilon_{j}, i^{\prime} \in I_{j}, i \neq i^{\prime}, n} \chi_{i, i^{\prime}, j, n} \geq-1+\sum_{s \in S_{j}^{p}, i \in I_{s}^{p}, i \in I_{j}} 1, \quad \forall j \in J^{M} \cap J^{B U}
\end{aligned}
$$

Here parameter $A m t^{\mathrm{min}}=\sum_{s \in S_{A}, o \in O_{S}} D_{o, s}^{-}-\sum_{s \in S_{A}, k \in K_{s}} s t o_{s, k}$. Constraint (49) enforces that tasks that produce product $s$ must be active at least once over the scheduling time horizon and constraint (50) enforces minimum number of changeover of tasks at multipurpose blend units. Constraint (49) is added to both (PSP) and (BSP); however, Eq. (50) is only present in (BSP).

$$
\begin{aligned}
\sum_{s \in S_{b c}, j \in \in_{s}^{p}, n \neq N} U o f_{s, j, n} \geq A m t^{\mathrm{min}}-\text { pen } \\
\operatorname{minimize}\left\{L^{P m}(u)=z+C^{11} p e n-\sum_{j \in J^{B U}, t} u_{j, t}^{T s} T s_{j, t}^{P}-\sum_{j \in J^{B U}, t} u_{j, t}^{T f} T f_{j, t}^{P}-\sum_{j \in J^{B U}, s \in S_{j}^{C}, t} u_{s, j, t}^{C f} C f_{s, j, t}^{P}\right\}
\end{aligned}
$$

Constraint (51) enforces that (PSP) must produce at least minimum amount of blend components required for (BSP) so that demand of blend products can be satisfied. If due to production capacity limitation, (PSP) cannot produce enough blend components, then we add positive penalty variable $p^{\text {en }}$ in Eq. (51) which is penalized in the objective function as shown in Eq. (52). The objective function of relaxed and restricted relaxed problems is now replaced by the one given in Eq. (52).

\subsubsection{Construction of lower bound from the best upper bound}

Due to production capacity limitation, the refinery might not be able to satisfy the demand of the products on time, might not respect all the blend logistics requirements, and may not be able to meet product quality specifications. To obtain a feasible schedule that can be implemented in the refinery, we have included, demand, due dates, and blend logistics giveaways in all scheduling models. These violations are heavily penalized in the objective function. Eq. (53a) gives the demand and due violations cost and Eq. (53b) gives total violation cost by including the product downgrading and minimum heel violation penalty to the demand violation cost. We also obtain the cost of task changeovers for multipurpose blend units from feasible solution as given in Eq. (53c).

$$
\begin{aligned}
& \text { violatn }_{\text {dem }}=\sum_{o \in O} c_{o}^{11} d g_{o}^{l}+\sum_{o \in O} c_{o}^{12} d g_{0}^{u}+\sum_{s \in S_{B}} c_{s}^{13} r g_{s}+\sum_{o \in O} c_{0}^{14} \text { Tearly }_{o}+\sum_{o \in O} c_{o}^{15} \text { Tlate }_{o} \\
& \text { violatn }_{\text {total }}=\text { violatn }_{\text {dem }}+\sum_{k \in K^{m}, s \in S_{k}, s^{\prime} \in S_{k}, s \neq s^{\prime}, n} c_{k \in K^{m}}^{9} s t d_{s, s^{\prime}, k, n}+\sum_{k \in K^{m}, s \in S_{k}, n} c_{s, k}^{10} m h_{s, k, n} \\
& \text { chgovr }=\sum_{j \in J^{m}, i \in I_{j}, i^{\prime} \in I_{j}, i^{\prime} \neq i, n} c_{i, i^{\prime}}^{6} \chi_{i, i^{\prime}, j, n}
\end{aligned}
$$

For a minimization problem, $Z^{L} \leq Z^{\text {opt }} \leq Z^{U}$ thus, if the best upper bound obtained at the current iteration provides a solution without any violations, then the optimal schedule of the original integrated problem will not have any violations. Furthermore, in refinery, an optimal solution with lowest task changeovers cost is desired if there are no demand and due-date violations. The desired changeovers at multipurpose blend units are from task producing high quality blend product to tasks producing low quality products so that the products produced during changeover transition are not discarded. Thus, we can conclude that chgovropt $^{\text {op }}$ chgovr $^{U}$, if violatn $_{\text {total }}^{U}=0$.

$$
\begin{aligned}
& \left\{d_{o}^{l}, d g_{o}^{u}, r g_{s}, \text { Tearly }_{o}, \text { Tlate }_{o}\right\}=0, \quad \text { if } \text { violatn }_{\text {dem }}^{U}=0 \\
& \left\{d g_{0}^{l}, \operatorname{dg}_{0}^{u}, \operatorname{rg}_{s}, \text { Tearly }_{0}, \text { Tlate }_{0}, s t d_{s, s^{\prime}, k, n}, m h_{s, k, n}\right\}=0, \quad \text { if } \text { violatn }_{\text {total }}^{U}=0 \\
& \sum_{j \in J^{m}, i \in I_{j}, i^{\prime} \in I_{j}, i^{\prime} \neq i, n} c_{i, i^{\prime}}^{6} \chi_{i, i^{\prime}, j, n} \leq \text { chgovr }^{U}, \quad \text { if } \text { violatn }_{\text {total }}^{U}=0
\end{aligned}
$$

We can produce tighter lower bound by considering value of parameter violatn $\mathrm{total}^{U}$ obtained from the best upper bound solution for a given set of Lagrangian multipliers. If best upper bound obtained satisfies finished products demand and their due-dates, violatndem $=0$, then we fixed variables associated with demands and due-dates to zero as given by Eq. (54a). Similarly, in constraint (54b), variables associated with demand and blend logistics violations are fixed to zero if the best upper bound solution where violatn $n_{\text {total }}^{U}=0$. Furthermore, total changeover cost for multipurpose blend units is bounded by the total changeover cost (chgovr ${ }^{U}$ ) using Eq. (54c). 


\subsection{Updating the multipliers}

The subgradient method first proposed by Fisher (1981) is commonly used method to update Lagrangian multipliers used in solving Lagrangian relaxation problems. It requires solving all sub-problems at each iteration to find a subgradient of the relaxed problem to compose a search direction to update the multipliers. The elements of subgradient to the relaxed problems are as follows:

$$
\begin{aligned}
& g_{j, t}^{T s}=\overline{T s}_{j, t}^{B}-\overline{T S}_{j, t}^{P}, \quad \forall j \in J^{B U}, t \in T \\
& g_{j, t}^{T f}=\overline{T f}_{j, t}^{B}-\overline{T f}_{j, t}^{P}, \quad \forall j \in J^{B U}, t \in T \\
& g_{s, j, t}^{C f}=\overline{C f}_{s, j, t}^{B}-\overline{C f}_{s, j, t}^{P}, \quad \forall j \in J^{B U}, s \in S_{j}^{c}, t \in T
\end{aligned}
$$

where $\left(\overline{C f}_{s, j, t}^{B}, \overline{T s}_{j, t}^{B}, \overline{T f}_{j, t}^{B}\right)$ and $\left(\overline{C f}_{s, j, t}^{P}, \overline{T s}_{j, t}^{P}, \overline{T f}_{j, t}^{P}\right)$ are the values of the duplicating variables, obtained from the solution of the blend scheduling problem and production scheduling problem, respectively.

The multiplier is updated for the next iteration according to

$$
u^{(m+1)}=u^{(m)}+s^{(m)} d^{(m)}
$$

Here, $u^{(m)}$ denotes the multiplier values, $s^{(m)}$ is the step size, and $d^{(m)}$ is the search direction in iteration $m$. The search direction is usually set to the subgradient, but it is more efficient to use modified formula that takes into consideration pervious iteration direction. Search direction is defined as recursive formula as suggested in Gaivoronski (1988), where $d^{(1)}=g^{(1)}$ :

$$
d^{(m)}=\frac{\left(g^{(m)}+\theta d^{(m-1)}\right)}{\theta+1}, \quad \forall m>1
$$

where $\theta$ determines how much consideration is given to the previous direction, 0 being no consideration to previous direction and 1 being an average of current and previous search direction. The step size $s^{(m)}$ is given by a widely used formula:

$$
s^{(m)}=\lambda^{(m)} \frac{\left(Z^{U}-L\left(u^{(m)}\right)\right)}{\left\|g^{(m)}\right\|^{2}}
$$

Here $Z^{U}$ is the upper bound of (IP), $L\left(u^{(m)}\right)$ is the solution of the relaxed problem at $u^{(m)}$, and $\lambda^{(m)}$ should be assigned a value in the interval $(\varepsilon$, $2-\varepsilon)$, where $\varepsilon>0$ to ensure convergence. If there is no improvement in the lower bound in $K$ successive iterations, we set $\lambda^{(m)}=0.5 \lambda^{(m-1)}$. $\lambda^{(m)}$ is reset back to $\lambda^{(0)}$ whenever an improved upper bound or lower bound.

\subsection{Stopping criteria}

In this section, we present the stopping criteria used to terminate the Lagrangian algorithm.

- Stop if the feasible solution is found at lower bound, which happens when $g^{(m)}=0$; but this rarely occurs for large problems.

- Stop if the lower bound from the Lagrangian relaxation exceeds the best known upper bound, i.e., if $Z^{L} \geq Z^{U}$.

- Stop if the duality gap is less than or equal to $1 \%$.

$$
\text { duality gap }=\left(\frac{Z^{U}-Z^{L}}{Z^{L}}\right)
$$

- Stop if $\left\|d^{(m)}\right\|<\varepsilon, s^{(m)}<\varepsilon$, or $Z^{U}-Z^{L} \leq \varepsilon$.

- In practice we do not wait till above mentioned stopping criteria is met, so we impose the number of iteration as the stopping criterion, i.e., stop after a limited number of iterations.

\section{Lagrangian decomposition algorithm}

In this section, proposed Lagrangian decomposition algorithm is presented in detail. This algorithm differs from classical Lagrangian decomposition in the way that the lower bound and Lagrange multipliers are obtained. In classical Lagrangian decomposition (classical-LD), the lower bound is always obtained from the Lagrangian relaxed problem where as in the proposed restricted Lagrangian decomposition (restricted-LD) the tighter lower bound is chosen among the relaxed problem and the restricted relaxed problem.

Before presenting the details of the algorithm, the scheduling models used in the algorithm are defined first. The full-scale model (IP) includes constraints (1)-(26). The relaxed blend scheduling model (P1) consists of tasks, units, materials, tanks relating to (BSP) and is defined as below:

$$
\operatorname{minimize}\left\{L^{B}(u)=z+\sum_{j \in J^{B U}, t} u_{j, t}^{T s} T s_{j, t}^{B}+\sum_{j \in J^{B U}, t} u_{j, t}^{T f} T f_{j, t}^{B}+\sum_{j \in J^{B U}, s \in S_{j}^{C}, t} u_{s, j, t}^{C f} C f_{s, j, t}^{B}\right\}
$$

s.t. constraints corresponding to finished product blending and delivery operations in model

(IP) and (31a-31g), (32a-32d), (34a-34b), (36-37), (48a-48e), (49-50), (54a-54c) 
The relaxed production unit scheduling model (P2) consists of tasks, units, materials, tanks relating to (PSP) and is defined as below:

$$
\operatorname{minimize}\left\{L^{P m}(u)=z+C^{11} p e n-\sum_{j \in \in^{B U}, t} u_{j, t}^{T s} T s_{j, t}^{P}-\sum_{j \in J^{B U}, t} u_{j, t}^{T f} T f_{j, t}^{P}-\sum_{j \in \in^{B U}, s \in S_{j}^{C}, t} u_{s, j, t}^{C f} C f_{s, j, t}^{P}\right\}
$$

s.t. constraints corresponding to production units scheduling operations in model (IP), (38a-38b), (39-32),

(33a-33b), (34a-34d), (37a-37c), (38a-38d), (39-40), (49), (51), (54a-54c)

The restricted relaxed models (P1-R) and (P2-R) are constructed by fixing binary variables using Eqs. (43a)-(43h) in models (P1) and (P2), respectively.

$$
\begin{aligned}
& \operatorname{minimize}\left\{L^{B}(u)=z+\sum_{j \in J^{B U}, t} u_{j, t}^{T s} T s_{j, t}^{B}+\sum_{j \in J^{B U}, t} u_{j, t}^{T f} T f_{j, t}^{B}+\sum_{j \in J^{B U}, s \in S_{j}^{C}, t} u_{s, j, t}^{C f} C f_{s, j, t}^{B}\right\} \\
& \text { s.t. constraints corresponding to finished product blending and delivery operations in model } \\
& \text { (IP) and }(31 a-31 g),(32 a-32 d),(34 a-34 b),(36-37),(48 a-48 e),(49-50),(54 a-54 c), \text { and }(43 a-43 h) \\
& \operatorname{minimize}\left\{L^{P m}(u)=z+C^{11} p e n-\sum_{j \in \mathbb{B}^{B U}, t} u_{j, t}^{T S} T s_{j, t}^{P}-\sum_{j \in J^{B U}, t} u_{j, t}^{T f} T f_{j, t}^{P}-\sum_{j \in J^{B U}, s \in S_{j}^{C}, t} u_{s, j, t}^{C f} C f_{s, j, t}^{P}\right\} \\
& \text { s.t. constraints corresponding to production units scheduling operations in model(IP), (38a - 38b), (39-32), } \\
& (33 a-33 b),(34 a-34 d),(37 a-37 c),(38 a-38 d),(39-40),(49),(51),(54 a-54 c) \text {, and }(43 a-43 h)
\end{aligned}
$$

If the restricted relaxed sub-problems are infeasible, then infeasibility would occur because demands cannot be met on time. In case of the infeasible restricted relaxed sub-problems, feasible restricted relaxed sub-problems (P1-RI) and (P2-RI) are obtained by eliminating Eqs. (54a)-(54c) from models (P1-R) and (P2-R). The upper bounding problem (IP-U) is obtained by fixing values of certain variables using Eqs. (44a)-(44d) in full-scale model (IP). The models (PC-s) and (PC-j), defined below, are obtained from (P2) by replacing its objective function by (45) and (46), respectively.

$$
\operatorname{minimize}\left\{z_{s}^{P P}=-100 \sum_{j \in P^{P U}, j \in J_{s}^{p}, n \in N} U o f_{s, j, n}\right\}, \quad \forall s \in S_{b c}
$$

s.t. constraints corresponding to production units scheduling operations in model

(IP), (38a-38b), (39-32), (33a-33b), (34a-34d), (37a-37c), (38a-38d), (39-40)

$$
\operatorname{minimize}\left\{z_{j}^{P P}=-100 \sum_{j^{\prime} \in J^{P U}, s \in S_{j}^{C} \cap S_{j^{\prime}}^{p}, n \in N} U o f_{s, j, n}\right\}, \quad \forall j \in J^{B U}
$$

s.t. constraints corresponding to production units scheduling operations in model

(IP), (38a-38b), (39-32), (33a-33b), (34a-34d), (37a-37c), (38a-38d), (39-40)

Here using $u^{(m)}$ at $m$ th iteration, the objective value of the relaxed problem and restricted relaxed problem is calculated from Eq, in (4b) and given as $Z_{R}\left(u^{(m)}\right)$ and $Z_{R R}\left(u^{(m)}\right)$ respectively. $Z^{(m)}$ is the objective value of the feasible solution through constructed heuristic at the $m$ th iteration.

The procedure for the restricted-LD algorithm:

Step 1: Solve pre-processing problems (PC-j) and (PC-s) as described in Section 4.3.1.

Step 2: Initialize $m=0 ; Z^{U}=+\infty ; Z^{L}=-\infty ;\left(u_{j, t}^{T S}\right)^{(0)}=0 ;\left(u_{j, t}^{T f}\right)^{(0)}=0 ;\left(u_{s, j, t}^{C f}\right)^{(0)}=0 ; \lambda^{(0)} ; \theta$

Step 3: Solve relaxed problem by decomposition that is solve independent problems (P1) and (P2).

Obtain $Z_{R},\left(\overline{C f}_{s, j, t}^{B}\right)_{R}$, violatn $_{\text {total }}^{R}$, and chgovr $r_{R}$.

Step 4: Pick primary sub-problem and construct a restricted relaxed sub-problem as described in Section 4.2.1. Solve the restricted sub-problems (P1-R) or (P2-R).

If the restricted relaxed sub-problem is infeasible, then solve corresponding feasibility sub-problem using model (P1-RI) or (P2-RI).

Obtain $Z_{R R},\left(\overline{C f}_{s, j, t}^{B}\right)_{R R}$, violatn $_{\text {total }}^{R R}$, and chgovr $r_{R R}$.

Step 5: If violatn total $\leq$ violatn $n_{\text {total }}^{R}$ and $Z_{R R}\left(u^{(m)}\right)>Z^{L}$, then $Z^{L}=Z_{R R}\left(u^{(m)}\right)$.

Otherwise, if violatn total $>$ violatt $n_{\text {total }}^{R}$ and $Z_{R}\left(u^{(m)}\right)>Z^{L}$, then $Z^{L}=Z_{R}\left(u^{(m)}\right)$.

Step 6: Construct a feasible solution to the original problem as described in Section 4.2.2.

If $Z^{m}<Z^{U}$, then $Z^{U}=Z^{m}$ and obtain parameters violatn dem violatn $_{\text {total }}^{U}$, and chgovr ${ }^{U}$ for constraints described in Section 4.3.3.

Step 7: Update Lagrangian multipliers by using the sub-gradient method presented in Section 4.4.

Step 8: Terminate the algorithm if the current solution satisfies at least one pre-defined stopping conditions listed in Section 4.5. Otherwise, set $m=m+1$ and return to step 3 .

In step 7, the Lagrangian multipliers are calculated using restricted relaxed problem solution unless violatn total ${ }^{R R}$ violatn $_{\text {total }}^{R}$. Problems in step 1 are independent and can be solved in parallel. Similarly, optimization models (P1) and (P2) in step 3 and models (P1-R) and (P2-R) 


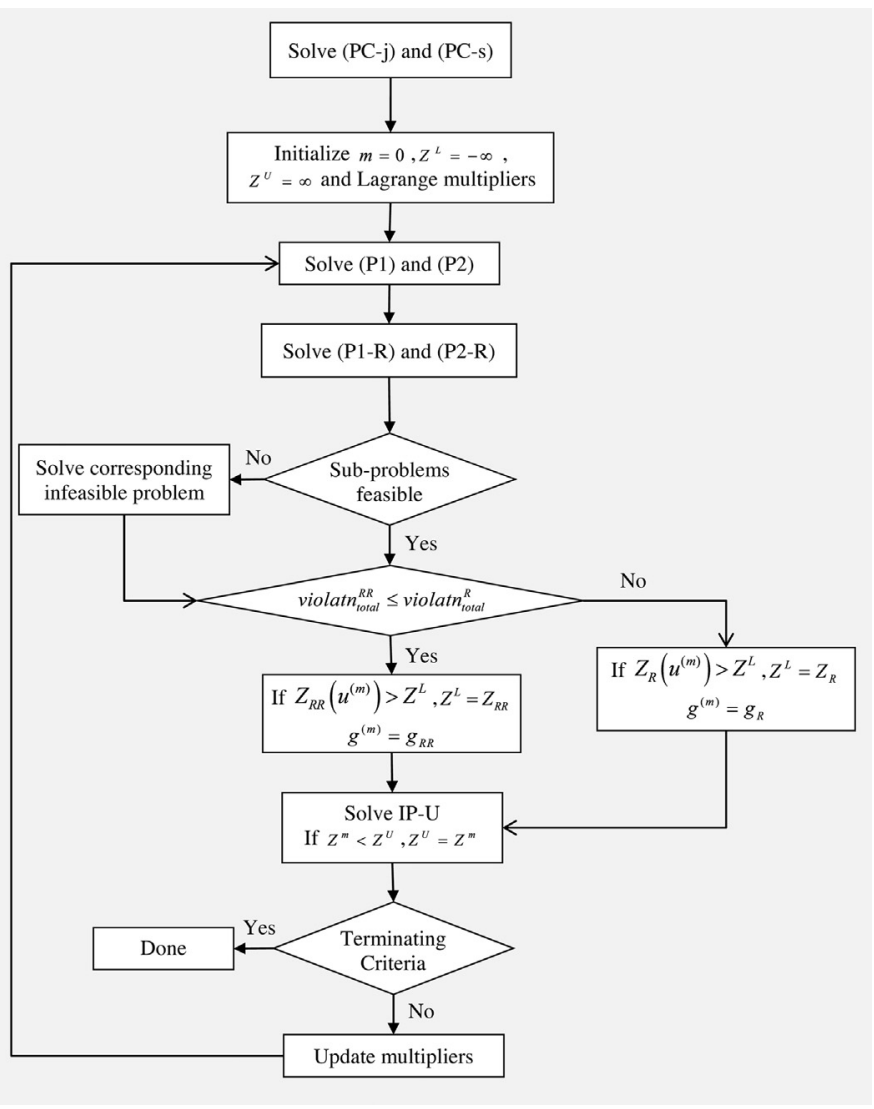

Fig. 5. Algorithm for the proposed restricted-Lagrangian decomposition (restricted-LD).

in step 4 are independent of each other and can be solve in parallel to reduce computational time. The flowchart of the framework for the algorithm is given in Fig. 5.

\section{Case study}

The refinery studied in this paper is a case study with realistic data provided by Honeywell Process Solutions (HPS). The refinery produces diesel fuels, jet fuel, and feed stocks for gasoline production and other chemical products. There are 2 blend units, 2 non-identical parallel crude distillation units (CDUs), and 13 other production units. The schematic of production system is shown in Fig. 6. There are two charging tanks, 5 intermediate tanks, and 9 product tanks. Jet blender unit blends three blend component streams to produce jet fuel that can be stored in 2 product tanks and diesel blender unit produces three different grades of fuel: CARB diesel, EPA diesel, and Red-dye diesel by blending three different components using three different run-modes. There are two dedicated tanks for each grade of diesel products and one multipurpose tank that can service CARB and EPA diesel. There is a $6 \mathrm{~h}$ of cleaning or maintenance downtime when the multipurpose tank service switches from lower grade of diesel product to a higher grade of product. This cleaning downtime is essential to remove any sulfur contamination present in the tank before low sulfur product is sent for storage. The product tank has $4 \mathrm{~h}$ of down time called fill-draw-delay for certificate of analysis preparation and to let the product settle down and mix before is shipped to the market. A minimum run-length restriction of $6 \mathrm{~h}$ is imposed on all production units.

To apply Lagrangian decomposition, we decompose the problem into blend scheduling problem and production unit scheduling problem. The blend scheduling problem includes two blend units (jet and diesel blenders). The two blend units mix different components and store blend products in tanks dedicated to each blend units. Thus, we can further decompose the blend scheduling problem into two subproblems, one problem includes the diesel blender and the products tanks associated with it, and another sub-problem includes the jet blender and the product tanks associated with it. The production unit scheduling problem cannot be decomposed further since there are interconnections between units that produce blend components for two different blend scheduling sub-problems. Case study data for production capacity, recipe, and storage tanks are provided in Supplementary material.

\section{Computational results}

The computational results are obtained on Dell Precision (IntelR XeonTM with CPU 3.20 GHz, 3.19 GHz, and 2 GB memory) running on Linux using CPLEX 12.3.0/GAMS 23.7.2 to demonstrate their effectiveness in solving oil-refinery scheduling problems using 6 different examples, where each example differs in either demands values, intermediate due dates, and initial hold up in the tank. The Lagrangian algorithm is set up in MATLAB and interfaces with GAMS to solve scheduling problems. The algorithm is evaluated with three performance measures: average duality gap, number of iterations, and computational time (seconds). The maximum solution time of $28,800 \mathrm{CPU}$ seconds is used for full-scale problem and for the decomposed problem maximum solution time of $3600 \mathrm{CPU}$ seconds is used. Optimality tolerance 


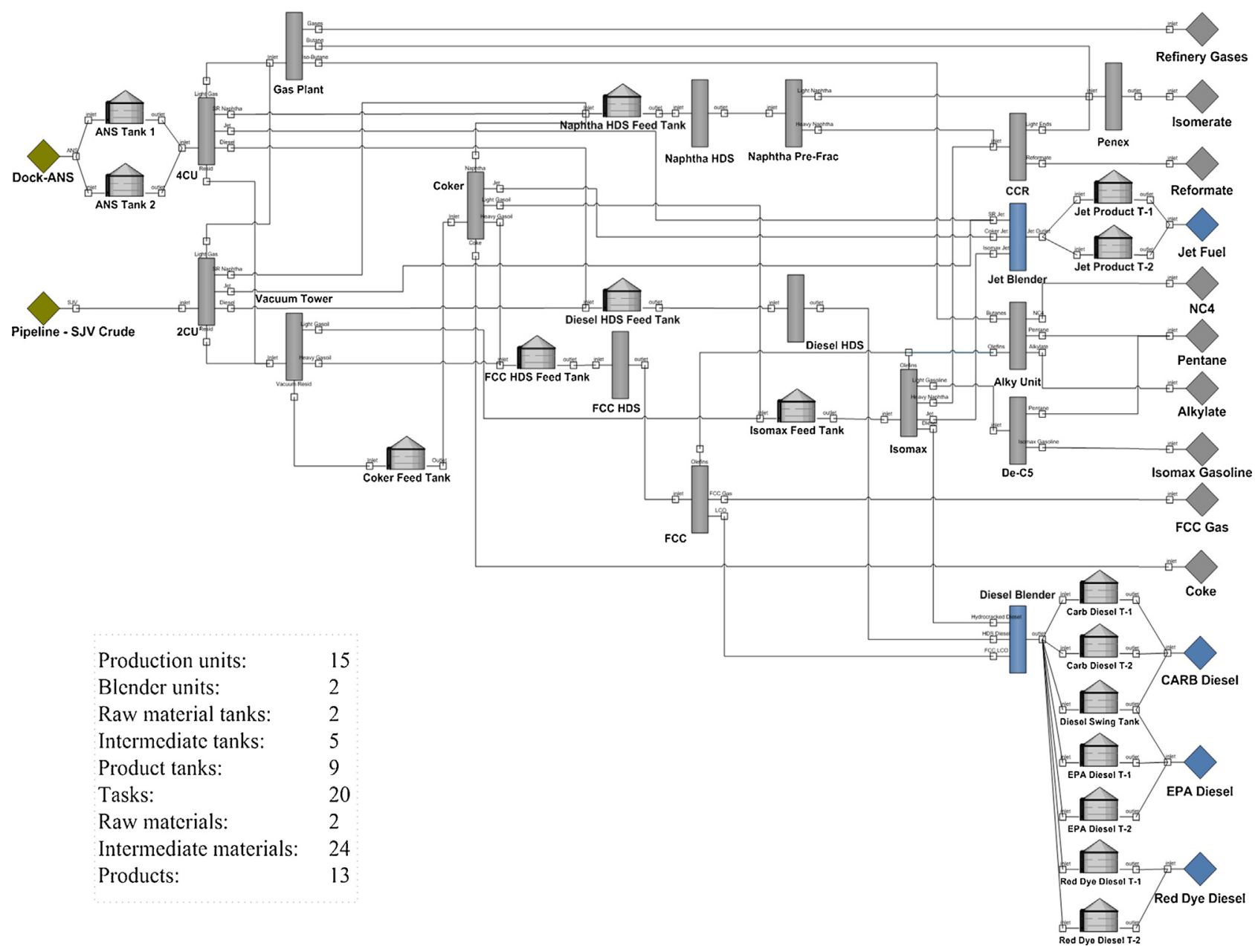

Fig. 6. Schematic of the refinery network.

Table 1

Penalty parameters in objective function.

\begin{tabular}{|c|c|c|c|}
\hline Penalty parameter & Value & Penalty parameter & Value \\
\hline$C_{\text {CarbNormal.DieselBlender }}^{1}$ & 10 & $C_{s, s^{\prime}}^{7}$ & 40 (unfavorable: 60 ) \\
\hline$C_{\text {EPANormal,DieselBlender }}^{1}$ & 7 & $C_{s, s^{\prime}}^{s, S^{\prime}}$ & 40 (unfavorable: 60 ) \\
\hline$C_{\text {ReddyeNormal,DieselBlender }}^{1}$ & 4 & $C_{k}^{9}$ & 5 \\
\hline$C_{\text {JetNormal,JetBlender }}^{1}$ & 5 & $C_{s, k}^{10}$ & 5 \\
\hline$C_{k}^{2}$ & 1 (swing tank: 3 ) & $C_{0}^{11}$ & 1150 \\
\hline$C_{k}^{3}$ & $50 \cdot\left(V_{k}^{\max }\right)^{-1}$ & $C_{o}^{12}$ & 800 \\
\hline$C_{j}^{4}$ & 50 & $C_{s}^{13}$ & 1100 \\
\hline$C_{k}^{5}$ & 10 (swing tank: 40) & $C_{o}^{14}$ & 1400 \\
\hline$C_{i, i^{\prime}}^{k}$ & 100 (unfavorable: 250 ) & $C_{o}^{15}$ & 1250 \\
\hline
\end{tabular}

of $1 \mathrm{e}-6$ is used as termination criteria for all examples solved using CPLEX. For the algorithm, the maximum number of iteration is set to $100, \varepsilon=1^{-6}$, and $K=2$. Penalty parameters for objective function is given in Table 1 and demand data for blend product and middle distillate product is given in Tables A1 and A2, respectively in Appendix.

The preprocessing problems for each blend components and blend units are solved before Lagrangian algorithm is implemented. The model statistics for preprocessing models are presented in Table 2 and these models are solved in less than 1 CPU seconds. The model

Table 2

Model statistics for preprocessing problems.

\begin{tabular}{|c|c|c|c|}
\hline & \multirow[t]{2}{*}{$(\mathrm{PC}-\mathrm{s})$} & \multicolumn{2}{|l|}{$(P C-j)$} \\
\hline & & Diesel blender & Jet blender \\
\hline Event pt. & 3 & 3 & 3 \\
\hline Int./Cont. var. & $120 / 924$ & $120 / 980$ & $120 / 978$ \\
\hline (Constraints) & $(2425)$ & $(2425)$ & $(2425)$ \\
\hline Nonzero Elem. & 7753 & 7813 & 7813 \\
\hline
\end{tabular}


Table 3

Model statistics.

\begin{tabular}{|c|c|c|c|c|c|}
\hline \multirow[t]{2}{*}{ Ex. } & \multirow[t]{2}{*}{ Orders } & \multirow[t]{2}{*}{$\begin{array}{l}\text { Full scale model } \\
\text { Event pt. } \\
\text { Int./Cont. var. } \\
\text { (Constraints) } \\
\text { Nonzero Elem. }\end{array}$} & \multicolumn{2}{|c|}{$\begin{array}{l}\text { Blend scheduling model (P1) } \\
\text { Event pt. } \\
\text { Int./Cont. variables } \\
\text { (Constraints) } \\
\text { Nonzero Elem. }\end{array}$} & \multirow[t]{2}{*}{$\begin{array}{l}\text { Production scheduling model } \\
\text { (P2) } \\
\text { Event pt. } \\
\text { Int./Cont. var. } \\
\text { (Constraints) } \\
\text { Nonzero Elem. }\end{array}$} \\
\hline & & & Diesel blender & Jet blender & \\
\hline 1 & 4 & $\begin{array}{l}5 \\
360 / 2663 \\
(6387) \\
22,633\end{array}$ & $\begin{array}{l}5 \\
127 / 958 \\
(2489) \\
9819\end{array}$ & $\begin{array}{l}5 \\
33 / 247 \\
(717) \\
2323\end{array}$ & $\begin{array}{l}5 \\
200 / 1526 \\
(4649) \\
15,394\end{array}$ \\
\hline 2 & 4 & $\begin{array}{l}5 \\
360 / 2663 \\
(6379) \\
22,569\end{array}$ & $\begin{array}{l}5 \\
127 / 958 \\
(2481) \\
9760\end{array}$ & $\begin{array}{l}5 \\
33 / 247 \\
(717) \\
2329\end{array}$ & $\begin{array}{l}5 \\
200 / 1526 \\
(4649) \\
15,405\end{array}$ \\
\hline 3 & 4 & $\begin{array}{l}5 \\
360 / 2663 \\
(6379) \\
22,569\end{array}$ & $\begin{array}{l}5 \\
127 / 958 \\
(2480) \\
9725\end{array}$ & $\begin{array}{l}5 \\
33 / 247 \\
(717) \\
2324\end{array}$ & $\begin{array}{l}5 \\
200 / 1526 \\
(4649) \\
15,395\end{array}$ \\
\hline 4 & 6 & $\begin{array}{l}5 \\
380 / 2771 \\
(6741) \\
24,018\end{array}$ & $\begin{array}{l}5 \\
139 / 1022 \\
(2695) \\
10,598\end{array}$ & $\begin{array}{l}5 \\
41 / 291 \\
(847) \\
2794\end{array}$ & $\begin{array}{l}5 \\
200 / 1526 \\
(4648) \\
15,399\end{array}$ \\
\hline 5 & 8 & $\begin{array}{l}6 \\
484 / 3453 \\
(8644) \\
31,765\end{array}$ & $\begin{array}{l}6 \\
184 / 1309 \\
(3649) \\
14,956\end{array}$ & $\begin{array}{l}6 \\
60 / 402 \\
(1272) \\
4384\end{array}$ & $\begin{array}{l}6 \\
240 / 1835 \\
(5794) \\
19,442\end{array}$ \\
\hline 6 & 13 & $\begin{array}{l}7 \\
736 / 5005 \\
(13,394) \\
53,145\end{array}$ & $\begin{array}{l}7 \\
277 / 1829 \\
(5585) \\
23,804\end{array}$ & $\begin{array}{l}7 \\
83 / 530 \\
(1833) \\
6643\end{array}$ & $\begin{array}{l}7 \\
280 / 2146 \\
(7027) \\
23,903\end{array}$ \\
\hline
\end{tabular}

Table 4

Computational Results.

\begin{tabular}{|c|c|c|c|c|c|c|c|c|c|c|c|}
\hline \multirow[t]{2}{*}{ Ex } & \multicolumn{3}{|c|}{ Full scale model } & \multicolumn{4}{|c|}{$\begin{array}{l}\text { Classical LD } \\
\lambda^{(0)}=1.50, \theta=0.8\end{array}$} & \multicolumn{4}{|c|}{$\begin{array}{l}\text { Restricted LD } \\
\lambda^{(0)}=1.20, \theta=0.7\end{array}$} \\
\hline & $z$ & Gap (\%) & CPU sec & $z$ & Gap (\%) & CPU sec & Total Iter. & $z$ & Gap (\%) & CPU sec & Total Iter. \\
\hline 1 & 450.77 & 0.00 & 342 & 450.77 & 29.34 & 652 & 43 & 450.77 & -0.21 & 113 & 11 \\
\hline 2 & 598.64 & 0.00 & 367 & 598.64 & 5.92 & 2133 & 57 & 622.91 & 0.15 & 251 & 9 \\
\hline 3 & 6222.54 & 0.00 & 8952 & 6222.54 & 1.49 & 15,304 & 71 & 6222.54 & 0.79 & 3245 & 14 \\
\hline 4 & 592.81 & 0.00 & 1535 & 602.33 & 25.22 & 1048 & 26 & 592.81 & 0.95 & 1225 & 40 \\
\hline 5 & 503.19 & 0.00 & 6458 & 504.25 & 12.62 & 1234 & 22 & 503.86 & 7.72 & 2652 & 20 \\
\hline 6 & 749.35 & 72.8 & 28,800 & 552.50 & 18.04 & 36,865 & 20 & 512.65 & -0.86 & 20,490 & 15 \\
\hline
\end{tabular}

statistics for full-scale integrated model and relaxed sub-problems for 6 different examples are shown in Table 3. Total number variables in restricted relaxed sub-problems, (P1-R) and (P2-R), are same as that of corresponding relaxed models because (P1-R) and (P2-R) are obtained by restricting solution space by including additional constraints and fixing values of certain binaries/continuous $0-1$ variables in corresponding relaxed models, (P1) and (P2).

The computational results for full-scale model and Lagrangian algorithm are shown in Table 4. Proposed restricted-Lagrangian decomposition algorithm is quite effective for these refinery scheduling problems and we obtain good solutions to the scheduling problem. Figs. 7-12 show the convergence of upper and lower bound using the classical LD and restricted LD algorithms in examples 1-6, respectively. The proposed restricted-LD algorithm outperforms classical Lagrangian decomposition in terms of quality of the solution, duality gap, number of iterations, and computational time. In classical approach, the lower bound is obtained solely from relaxed problem's solution, whereas proposed restricted-LD picks the best lower bound between the solution of relaxed problem and restricted relaxed problem. Thus, restricted-LD lower bounds take into consideration the continuous process characteristic of the refinery units and gives better lower bound as observed in Figs. 7-12.

In most cases, both algorithms provide an upper bound closer to the optimal solution of original problem in first iteration because feasible problem is constructed using restricted relaxed problem solutions and has flexibility of obtaining a better solution because only the certain binaries are fixed to 1 and none are fixed to 0 . In situation where first iteration does not provide an upper bound closer to optimal solution, the upper bound improves vastly early on and ultimately provides a better solution. In example 5, the first iteration provides an upper bound that is very far from an optimal solution, however, it improves greatly in second iteration, and by fifth iterations it is almost closed to the optimal value. After finding the best upper bound early on, both algorithms spend rest of the computational time proving optimality.

The performance (duality gap) of restricted-LD algorithm is better than that of classical approach because restricted-LD is able to improve lower bound faster than classical LD. Proposed restricted-LD provides better duality gap than classical LD and restricted-LD is terminated in almost all cases when gap is less than $1 \%$ as shown in Table 4 . In case of classical decomposition approach, the algorithm was terminated for all examples when the step size meets the predefined tolerance of $1 \mathrm{e}^{-6}$. The step size reaches the tolerance faster because 


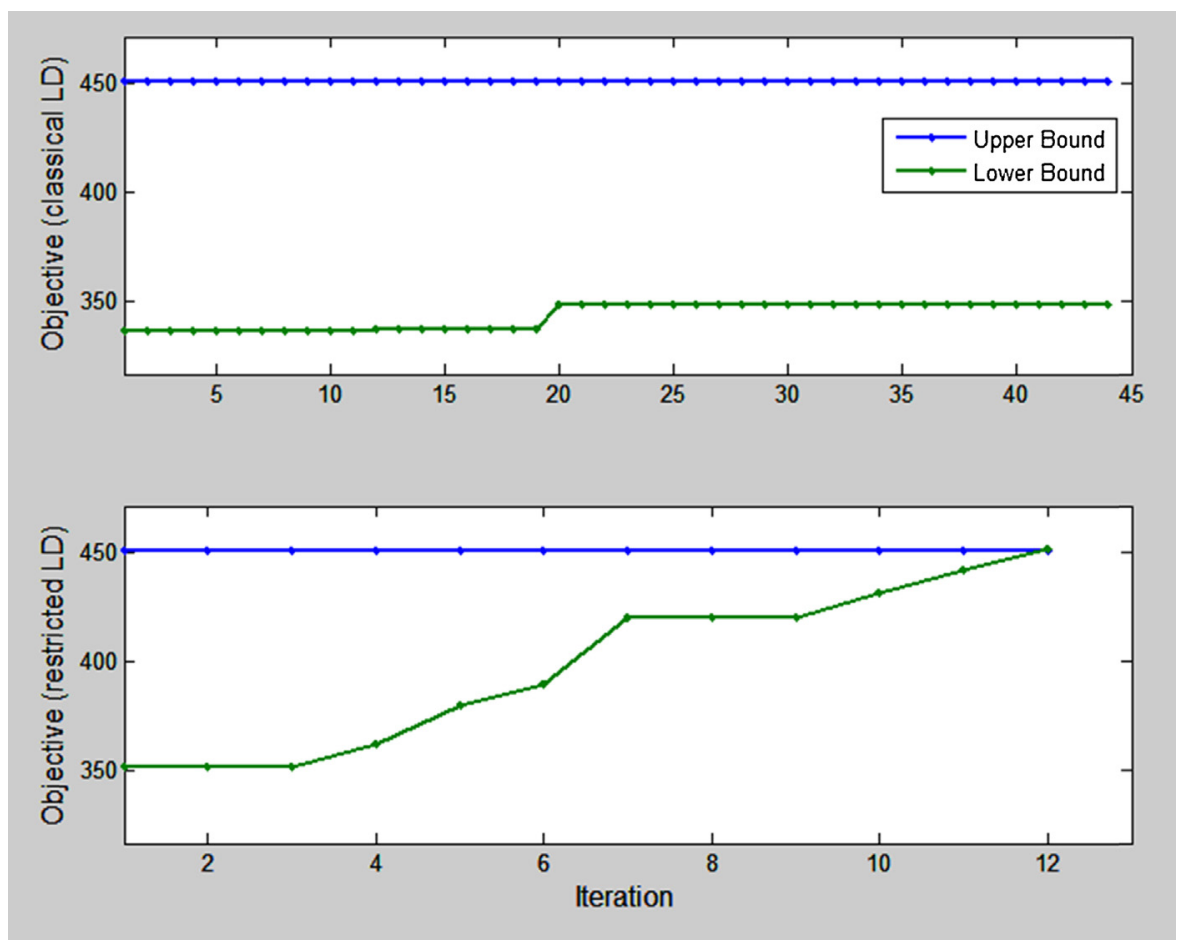

Fig. 7. Convergence of upper and lower bound of Lagrangian decomposition for example 1.

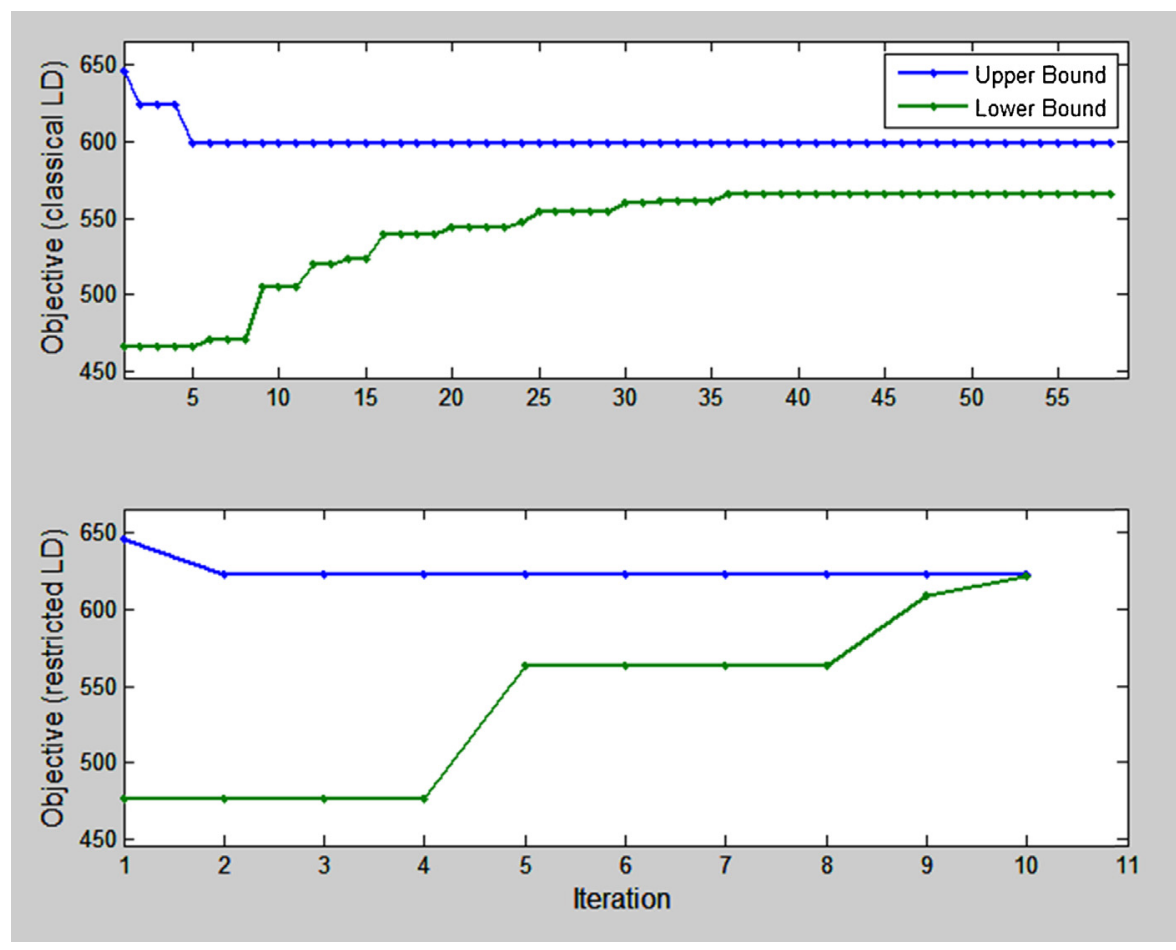

Fig. 8. Convergence of upper and lower bound of Lagrangian decomposition for example 2.

we cut the step size parameter $\lambda^{(m)}$ by half if the lower bound does not improve after 2 iterations. Even when the duality gap is high, the upper bound is closer to the original problem optimal solution in both algorithms.

Table 5 compares the computational effort exerted to solve relaxed problem, restricted relaxed problem, and feasible upper bounding problem. As expected, majority of the time is spent in solving relaxed problem. Classical LD takes 652 CPU seconds to solve example 1 and spends $77.32 \%$ of the total time on solving for relaxed problem and only $11.48 \%$ on solving restricted relaxed problem. Similarly for restricted-LD, example 1 takes $68.12 \%$ of the total computational time on solving relaxed problem, 13.26\% on solving restricted relaxed problem, and $18.63 \%$ on solving upper bounding problem MILP problem. Even for the large scale complex problem given in example 6 , 

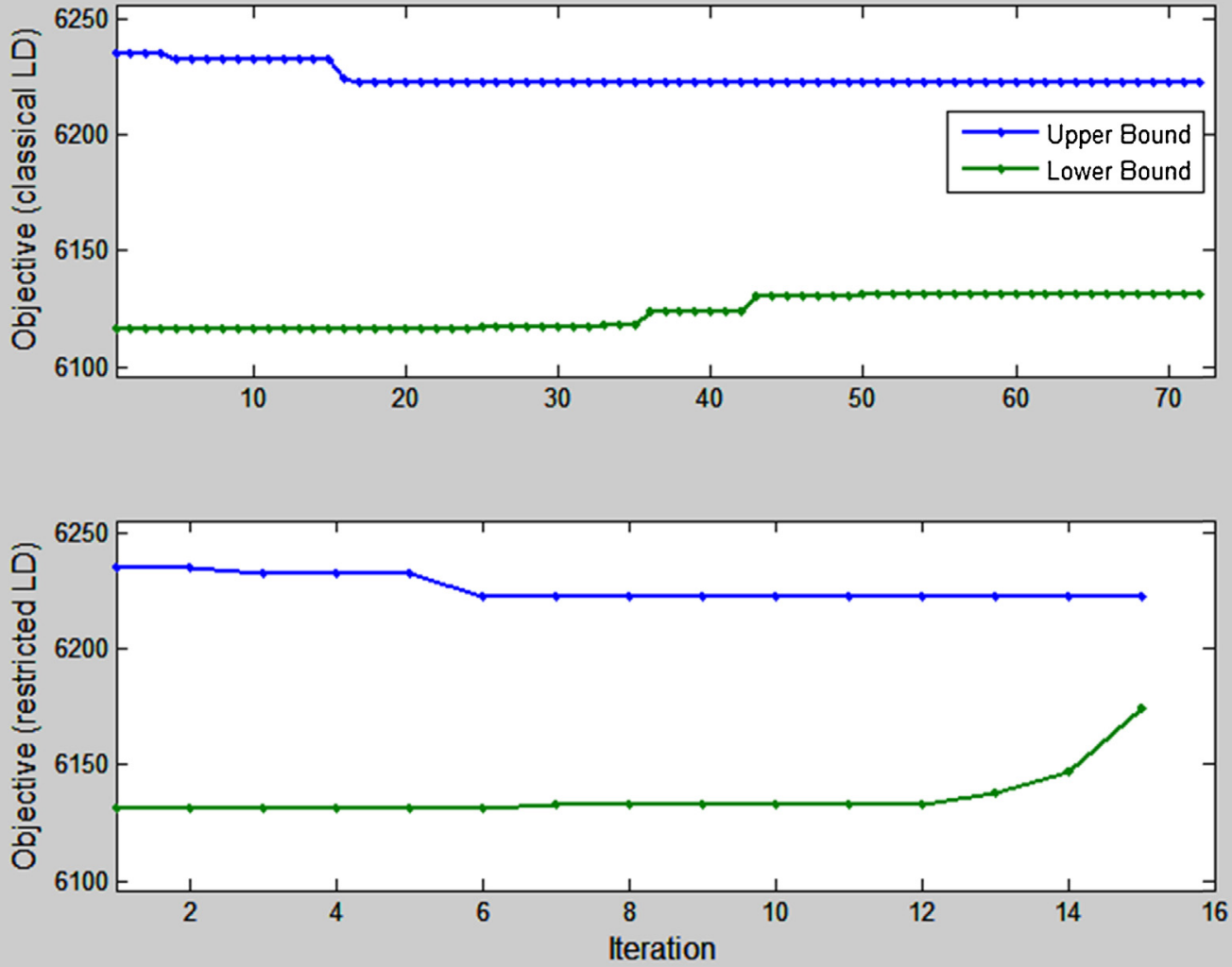

Fig. 9. Convergence of upper and lower bound of Lagrangian decomposition for example 3.

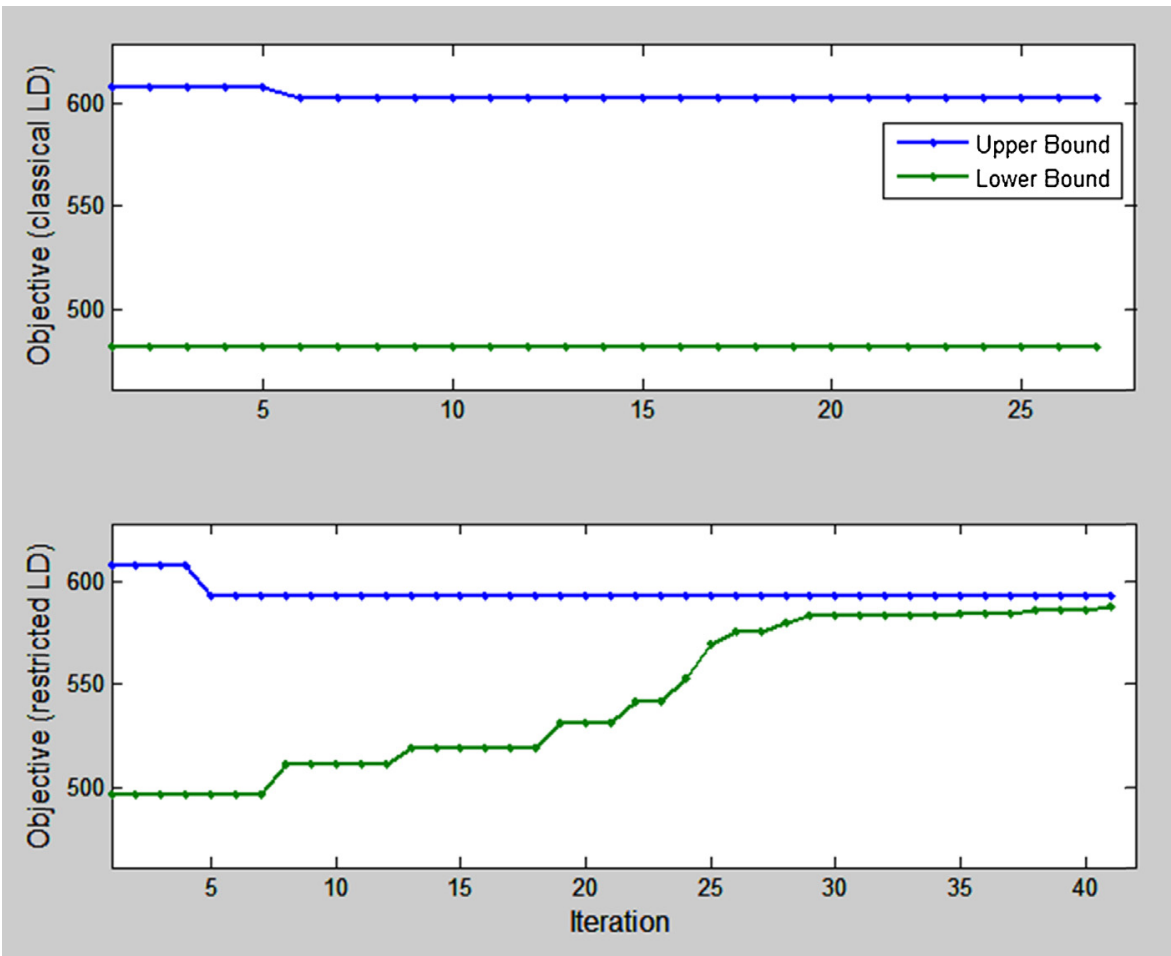

Fig. 10. Convergence of upper and lower bound of Lagrangian decomposition for example 4.

Please cite this article in press as: Shah NK, Ierapetritou MG. Lagrangian decomposition approach to scheduling large-scale refinery operations. Computers and Chemical Engineering (2015), http://dx.doi.org/10.1016/j.compchemeng.2015.04.021 


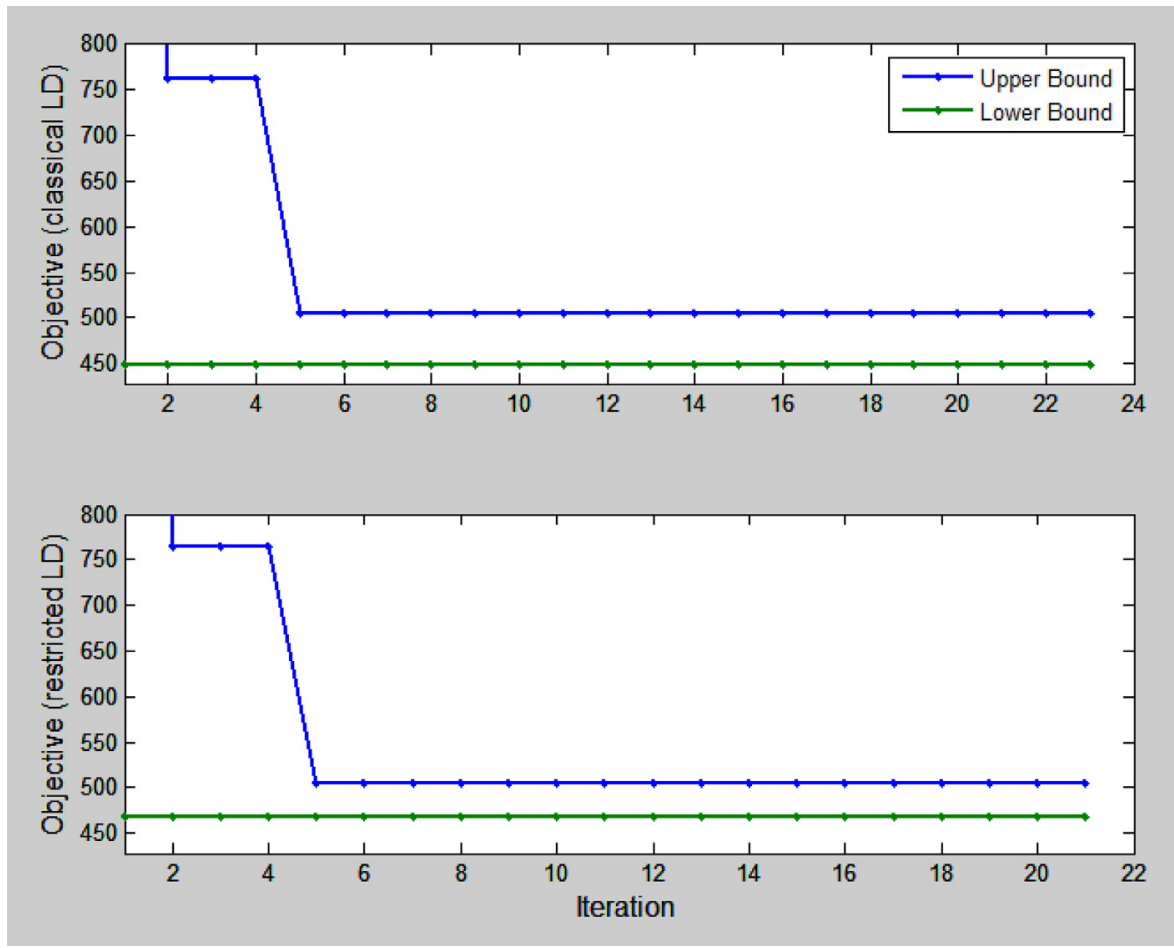

Fig. 11. Convergence of upper and lower bound of Lagrangian decomposition for example 5.

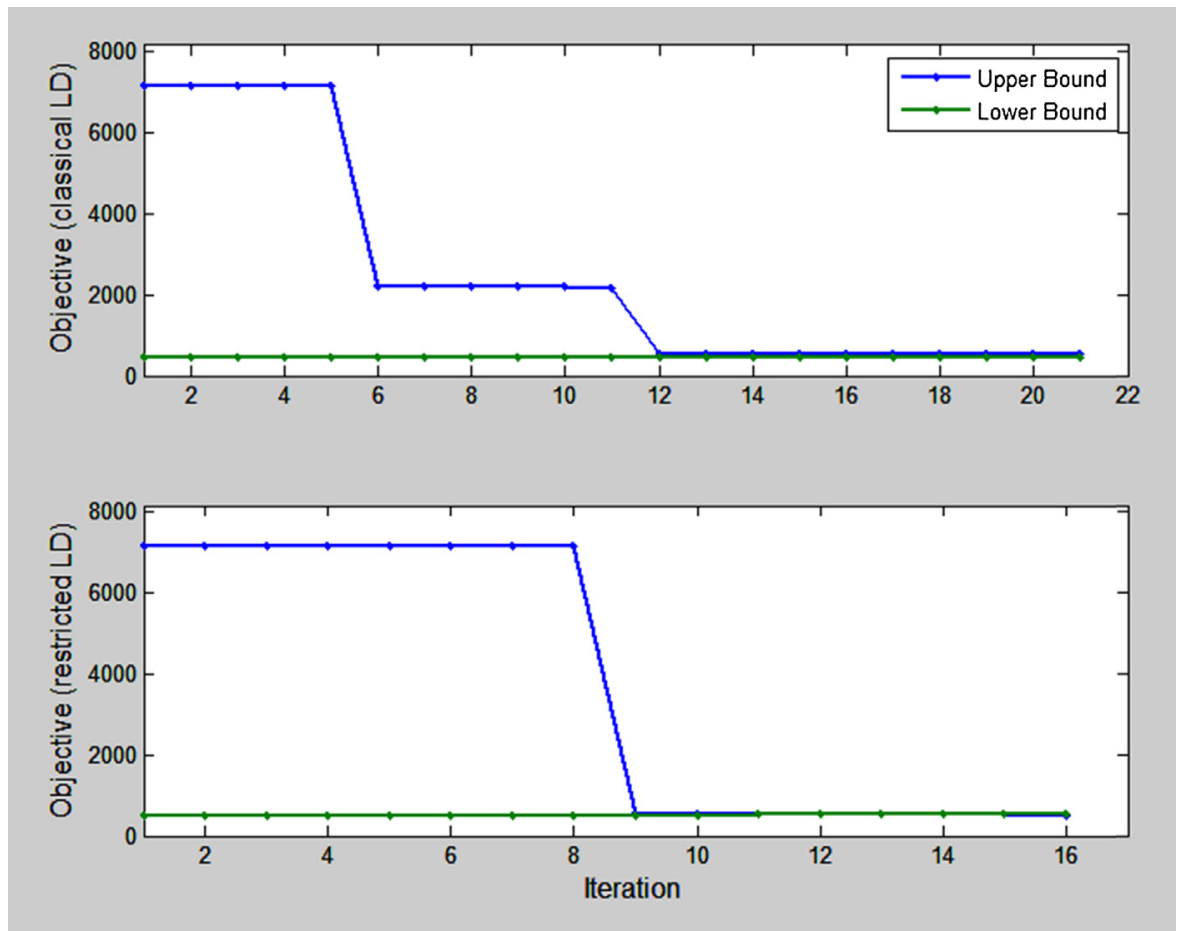

Fig. 12. Convergence of upper and lower bound of Lagrangian decomposition for example 6 .

Table 5

Time spent (\%) in each step compared to the total solution time of Lagrangian decomposition algorithms.

\begin{tabular}{|c|c|c|c|c|c|c|c|}
\hline & Problems & Example 1 & Example 2 & Example 3 & Example 4 & Example 5 & Example 6 \\
\hline \multirow[t]{3}{*}{ Classical LD } & Relaxed & 77.32 & 91.47 & 98.28 & 90.78 & 94.16 & 93.31 \\
\hline & Restricted relaxed & 11.48 & 4.40 & 0.96 & 4.17 & 2.80 & 2.95 \\
\hline & Feasible & 11.20 & 4.13 & 0.75 & 5.05 & 3.04 & 3.74 \\
\hline Restricted & Relaxed & 68.12 & 87.92 & 98.76 & 89.61 & 97.48 & 91.96 \\
\hline \multirow[t]{2}{*}{ LD } & Restricted relaxed & 13.26 & 4.94 & 0.49 & 4.19 & 1.31 & 7.96 \\
\hline & Feasible & 18.63 & 7.14 & 0.75 & 6.19 & 1.21 & 0.076 \\
\hline
\end{tabular}


the upper bounding MILP problem can be easily solved. Computational time can be further improved by solving relaxed sub-problems in parallel and similarly restricted relaxed problems can be solved in parallel.

\section{Summary}

This paper introduces a Lagrangian decomposition framework, restricted-LD for integrated production unit scheduling and finished product blending and delivery scheduling problems. The algorithm is built based on the mathematical formulation given by Shah and Ierapetritou (2011). A restricted relaxed problem is constructed based upon the solution of relaxed sub-problems to take into consideration the continuous process characteristic of the refinery. To improve the performance of the algorithm, a preprocessing step, constraints for decomposed sub-problems, and inclusion of the best upper bound's solution in lower bounding problems are proposed. The proposed restricted relaxed sub-problems produce better lower bounds and better upper bounds. Computational results of a real case study show that the proposed algorithm is very effective and provide better solutions in reasonable times.

\section{Acknowledgment}

The authors gratefully acknowledge support from the National Science Foundation through grant NSF-CBET 0966861 and GAANN.

Appendix.

Table A1

Group A products demand order data for the case study.

\begin{tabular}{|c|c|c|c|c|c|c|c|c|c|c|}
\hline \multirow[t]{2}{*}{ Orders } & \multicolumn{10}{|c|}{ Orders (product type, amount ( $\mathrm{kbbl})$, delivery window, delivery rate $(\mathrm{kbbl} / \mathrm{h})$ ) } \\
\hline & Ex1 & Ex2 & Ex3 & Ex4 & Ex5 & Ex6 & Ex7 & Ex8 & Ex9 & Ex10 \\
\hline \multirow[t]{3}{*}{1} & P3 & P3 & P2 & P3 & P1 & P3 & P1 & P3 & P1 & P2 \\
\hline & {$[60,80]$} & {$[50,70]$} & {$[100,120]$} & {$[90,100]$} & {$[120,125]$} & {$[60,75]$} & {$[25,30]$} & {$[15,25]$} & {$[40,50]$} & {$[20,55]$} \\
\hline & {$[30,50]$} & {$[20,35]$} & {$[15,25]$} & {$[50,75]$} & {$[40,75]$} & {$[28.5,46.5]$} & {$[12,30]$} & {$[30,50]$} & {$[30,40]$} & $20,35]$ \\
\hline \multirow[t]{3}{*}{2} & P2 & P4 & P4 & P4 & P2 & P2 & P1 & P2 & P2 & $\mathrm{P} 2$ \\
\hline & {$[50,70]$} & {$[50,90]$} & {$[50,90]$} & {$[140,150]$} & {$[100,110]$} & {$[90,100]$} & {$[50,65]$} & {$[40,50]$} & {$[40,55]$} & {$[35,50]$} \\
\hline & {$[60,90]$} & {$[45,80]$} & {$[45,80]$} & {$[60,90]$} & {$[90,115]$} & {$[65,89]$} & {$[50,70]$} & {$[45,75]$} & {$[45,58]$} & {$[40,55]$} \\
\hline \multirow[t]{3}{*}{3} & P4 & P2 & P3 & P1 & P1 & P4 & $\mathrm{P} 2$ & P4 & P1 & P1 \\
\hline & {$[50,80]$} & {$[50,75]$} & {$[50,75]$} & {$[100,115]$} & {$[150,175]$} & {$[140,150]$} & {$[45,50]$} & {$[50,75]$} & {$[45,53]$} & {$[40,50]$} \\
\hline & {$[75,100]$} & {$[60,80]$} & {$[60,80]$} & {$[100,120]$} & {$[135,150]$} & {$[75,91]$} & {$[90,105]$} & {$[75,110]$} & {$[70,85]$} & {$[60,75]$} \\
\hline \multirow[t]{3}{*}{4} & P1 & P1 & P1 & P4 & P2 & P1 & P4 & P2 & P3 & P3 \\
\hline & {$[50,75]$} & {$[50,80]$} & {$[50,80]$} & {$[100,120]$} & {$[90,120]$} & {$[90,115]$} & {$[90,110]$} & {$[20,30]$} & {$[40,44]$} & {$[15,60]$} \\
\hline & {$[75,110]$} & {$[70,90]$} & {$[70,90]$} & {$[125,150]$} & {$[168,185]$} & {$[102,116]$} & {$[98,128]$} & {$[75,95]$} & {$[92,105]$} & {$[80,100]$} \\
\hline \multirow[t]{3}{*}{5} & & & & $\mathrm{P} 2$ & P3 & P4 & P3 & P3 & $\mathrm{P} 2$ & $\mathrm{P} 2$ \\
\hline & & & & {$[110,125]$} & {$[105,125]$} & {$[100,150]$} & {$[50,65]$} & {$[10,20]$} & {$[30,60]$} & {$[30,50]$} \\
\hline & & & & {$[130,150]$} & {$[200,220]$} & {$[105,130]$} & {$[125,141]$} & {$[90,125]$} & {$[110,125]$} & {$[105,115]$} \\
\hline \multirow[t]{3}{*}{6} & & & & P1 & $\mathrm{P} 4$ & P1 & P2 & P1 & P3 & P3 \\
\hline & & & & {$[50,60]$} & {$[120,150]$} & {$[100,115]$} & {$[35,40]$} & {$[15,30]$} & {$[35,50]$} & {$[40,45]$} \\
\hline & & & & {$[160,175]$} & {$[50,75]$} & {$[125,153]$} & {$[160,180]$} & {$[100,115]$} & {$[120,140]$} & {$[110,125]$} \\
\hline \multirow[t]{3}{*}{7} & & & & & P4 & P4 & P4 & P1 & P1 & $\mathrm{P} 1$ \\
\hline & & & & & {$[100,120]$} & {$[100,165]$} & {$[100,115]$} & {$[55,60]$} & {$[35,40]$} & {$[30,65]$} \\
\hline & & & & & {$[120,146]$} & {$[155,170]$} & {$[160,193]$} & {$[115,145]$} & {$[145,160]$} & {$[130,145]$} \\
\hline \multirow[t]{3}{*}{8} & & & & & P4 & P2 & P3 & P4 & P3 & P1 \\
\hline & & & & & {$[160,170]$} & {$[75,100]$} & {$[45,65]$} & {$[75,100]$} & {$[30,75]$} & {$[25,50]$} \\
\hline & & & & & {$[175,205]$} & {$[179,203]$} & {$[175,205]$} & {$[115,145]$} & {$[175,190]$} & {$[150,165]$} \\
\hline \multirow[t]{3}{*}{9} & & & & & & P4 & P4 & P4 & P2 & P2 \\
\hline & & & & & & {$[120,125]$} & {$[85,100]$} & {$[80,100]$} & {$[40,40]$} & {$[30,45]$} \\
\hline & & & & & & {$[195,210]$} & {$[200,219]$} & {$[150,175]$} & {$[195,210]$} & {$[170,185]$} \\
\hline \multirow[t]{3}{*}{10} & & & & & & P3 & P1 & $\mathrm{P} 2$ & $\mathrm{P} 1$ & P3 \\
\hline & & & & & & {$[75,100]$} & {$[70,90]$} & {$[50,65]$} & {$[55,75]$} & {$[25,55]$} \\
\hline & & & & & & {$[200,215]$} & {$[210,227]$} & {$[155,170]$} & {$[205,220]$} & {$[170,190]$} \\
\hline \multirow[t]{3}{*}{11} & & & & & & & P2 & P3 & $\mathrm{P} 2$ & P1 \\
\hline & & & & & & & {$[50,65]$} & {$[10,15]$} & {$[40,45]$} & {$[20,55]$} \\
\hline & & & & & & & {$[220,240]$} & {$[160,180]$} & {$[205,225]$} & {$[200,220]$} \\
\hline \multirow[t]{3}{*}{12} & & & & & & & P4 & P3 & P1 & P1 \\
\hline & & & & & & & {$[120,140]$} & {$[35,40]$} & {$[50,60]$} & {$[25,45]$} \\
\hline & & & & & & & {$[223,240]$} & {$[180,200]$} & {$[220,240]$} & {$[215,235]$} \\
\hline \multirow[t]{3}{*}{13} & & & & & & & P3 & P2 & P4 & P3 \\
\hline & & & & & & & {$[60,80]$} & {$[10,15]$} & {$[50,75]$} & {$[30,40]$} \\
\hline & & & & & & & {$[228,240]$} & {$[200,220]$} & {$[50,75]$} & {$[220,240]$} \\
\hline \multirow[t]{3}{*}{14} & & & & & & & & P4 & P4 & P4 \\
\hline & & & & & & & & {$[100,115]$} & {$[75,115]$} & {$[40,60]$} \\
\hline & & & & & & & & {$[200,230]$} & {$[100,120]$} & {$[30,65]$} \\
\hline \multirow[t]{3}{*}{15} & & & & & & & & P1 & P4 & P4 \\
\hline & & & & & & & & {$[30,45]$} & {$[60,110]$} & {$[55,95]$} \\
\hline & & & & & & & & {$[210,235]$} & {$[125,150]$} & {$[55,75]$} \\
\hline
\end{tabular}


Table A1 (Continued)

\begin{tabular}{|c|c|c|c|c|c|c|c|c|c|c|}
\hline \multirow[t]{2}{*}{ Orders } & \multicolumn{10}{|c|}{ Orders (product type, amount (kbbl), delivery window, delivery rate (kbbl/h)) } \\
\hline & Ex1 & Ex2 & Ex3 & Ex4 & Ex5 & Ex6 & Ex7 & Ex8 & Ex9 & Ex10 \\
\hline 16 & & & & & & & & $\begin{array}{l}\text { P4 } \\
{[75,100]} \\
{[220,240]}\end{array}$ & $\begin{array}{l}\text { P4 } \\
{[55,120]} \\
{[140,165]}\end{array}$ & $\begin{array}{l}\text { P4 } \\
{[50,65]} \\
{[95,110]}\end{array}$ \\
\hline 17 & & & & & & & & & $\begin{array}{l}\text { P4 } \\
{[85,125]} \\
{[175,200]}\end{array}$ & $\begin{array}{l}\text { P4 } \\
{[30,105]} \\
{[115,125]}\end{array}$ \\
\hline 18 & & & & & & & & & $\begin{array}{l}\text { P4 } \\
{[100,125]} \\
{[205,225]}\end{array}$ & $\begin{array}{l}\text { P4 }[60,125] \\
{[130,155]}\end{array}$ \\
\hline 19 & & & & & & & & & $\begin{array}{l}\text { P4 } \\
{[78,100]} \\
{[220,240]}\end{array}$ & $\begin{array}{l}\text { P4 } \\
{[75,105]} \\
{[160,180]}\end{array}$ \\
\hline 20 & & & & & & & & & & $\begin{array}{l}\text { P4 } \\
{[85,115]} \\
{[180,205]}\end{array}$ \\
\hline 21 & & & & & & & & & & $\begin{array}{l}\text { P4 } \\
{[100,120]} \\
{[200,225]}\end{array}$ \\
\hline 22 & & & & & & & & & & $\begin{array}{l}\text { P4 } \\
{[110,130]} \\
{[215,240]}\end{array}$ \\
\hline
\end{tabular}

Table A2

Group B products demands data for the case study.

\begin{tabular}{|c|c|c|c|c|c|c|c|c|c|c|}
\hline \multirow[t]{2}{*}{ Ex. } & \multirow[t]{2}{*}{ Initial holdup in swing tank (product, kbbl) } & \multicolumn{9}{|c|}{ Group B products demand (kbbl) } \\
\hline & & P5 & P6 & P7 & P8 & P9 & P10 & P11 & P12 & P13 \\
\hline 1 & - & 5 & 5 & 10 & 10 & 10 & 5 & 4 & 5 & 5 \\
\hline 2 & P1-10 & 10 & 5 & 10 & 15 & 10 & 10 & 5 & 15 & 10 \\
\hline 3 & P1-10 & 5 & 10 & 15 & 10 & 5 & 5 & 10 & 25 & 5 \\
\hline 4 & - & 10 & 25 & 25 & 20 & 5 & 10 & 10 & 15 & 0 \\
\hline 5 & - & 15 & 30 & 25 & 25 & 15 & 13 & 10 & 50 & 10 \\
\hline 6 & - & 13 & 25 & 30 & 10 & 20 & 15 & 17 & 75 & 5 \\
\hline 7 & P1-10 & 10 & 20 & 30 & 25 & 30 & 15 & 10 & 60 & 15 \\
\hline 8 & - & 20 & 15 & 25 & 20 & 25 & 10 & 15 & 95 & 20 \\
\hline 9 & P1-10 & 15 & 35 & 35 & 20 & 20 & 15 & 20 & 70 & 10 \\
\hline 10 & - & 10 & 25 & 35 & 25 & 15 & 16 & 10 & 80 & 10 \\
\hline
\end{tabular}

\section{Appendix B. Supplementary data}

Supplementary data associated with this article can be found, in the online version, at http://dx.doi.org/10.1016/j.compchemeng.2015. 04.021

\section{References}

Adhya N, Tawarmalani M, Sahinidis NV. A Lagrangian approach to the pooling problem. Ind Eng Chem Res 1999;38:1956-72.

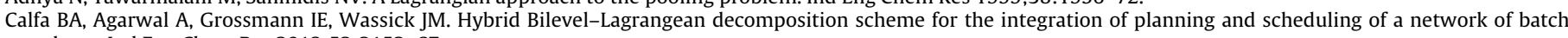
plants. Ind Eng Chem Res 2013;52:2152-67.

Castillo PAC, Mahalec V. Inventory pinch based, multiscale models for integrated planning and scheduling - part I: gasoline blend planning. AIChE J 2014a:60:2158-78.

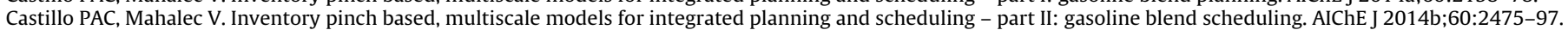

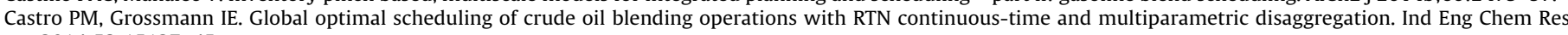
2014:53:15127-45

Fisher ML. The Lagrangian relaxation method for solving integer programming problems. Manage Sci 1981;27:1-18.

Gaivoronski A. Stochastic quasigradient methods and their implementation. Numerical techniques for stochastic optimization, vol. 10. Springer; 1988 . p. 313-51.

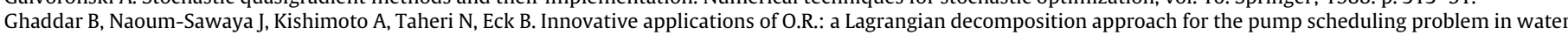
networks. Eur J Oper Res 2014.

Glismann K, Gruhn G. Short-term scheduling and recipe optimization of blending processes. Comput Chem Eng 2001;25:627-34

Graves SC. Using Lagrangian techniques to solve hierarchical production planning problems. Manage Sci 1982;28:260-75.

Grossmann IE. Advances in mathematical programming models for enterprise-wide optimization. Comput Chem Eng 2012;47:2-18.

Gupta A, Maranas CD. A hierarchical Lagrangian relaxation procedure for solving midterm planning problems. Ind Eng Chem Res 1999;38:1937-47.

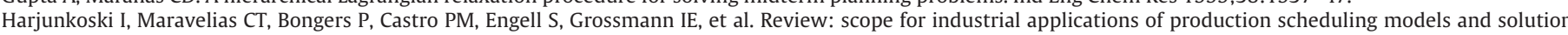
methods. Comput Chem Eng 2014;62:161-93.

Held M, Karp RM. The traveling-salesman problem and minimum spanning trees. Oper Res 1970;18:1138-62.

Holmberg K, Hellstrand J. Solving the uncapacitated network design problem by a Lagrangian heuristic and branch-and-bound. Oper Res 1998;46:247-59.

Holmberg K, Yuan D. A Lagrangian heuristic based branch-and-bound approach for the capacitated network design problem. Oper Res $2000 ; 48: 461-81$.

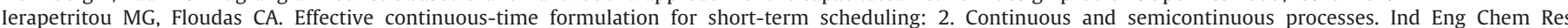
1998a;37:4360-74.

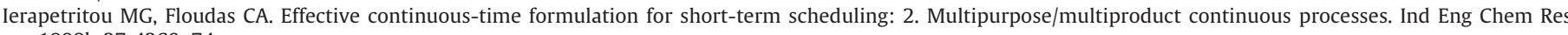
1998b;37:4360-74.

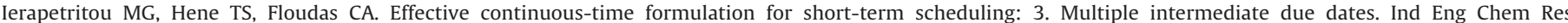
1999;38:3446-61.

Jia Z, Ierapetritou M. Mixed-integer linear programming model for gasoline blending and distribution scheduling. Ind Eng Chem Res 2003;42:825-35

Jia Z, Ierapetritou M. Efficient short-term scheduling of refinery operations based on a continuous time formulation. Comput Chem Eng 2004;28:1001-19. 
Joly M, Moro L, Pinto JM. Planning and scheduling for petroleum refineries using mathematical programming. Braz J Chem Eng 2002;19:207-28. Karuppiah R, Furman KC, Grossmann IE. Global optimization for scheduling refinery crude oil operations. Comput Chem Eng 2008;32:2745-66. Kelly JD. Next-generation refinery scheduling technology. In: NPRA plant automation and decision support conference; 2003, Presentation.

Kelly JD. Logistics: the missing link in blend scheduling optimization. Hydrocarb Process 2006:45-51.

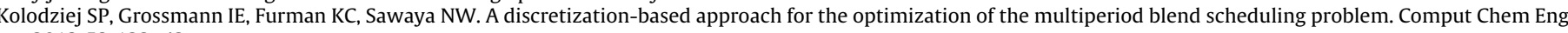
2013;53:122-42

Kondili E, Pantelides CC, Sargent RWH. A general algorithm for short-term scheduling of batch operations - I. MILP formulation. Comput Chem Eng 1993;17:211-27.

Li J, Karimi IA. Scheduling gasoline blending operations from recipe determination to shipping using unit slots. Ind Eng Chem Res 2011;50:9156-74.

Li Z, Ierapetritou MG. Production planning and scheduling integration through augmented Lagrangian optimization. Comput Chem Eng 2010;34:996-1006.

Luh PB, Hoitomt DJ. Scheduling of manufacturing systems using the Lagrangian relaxation technique. IEEE Trans Autom Control 1993;38:1066-79.

Luo C, Rong G. Hierarchical approach for short-term scheduling in refineries. Ind Eng Chem Res 2007;46(4474):3656-68.

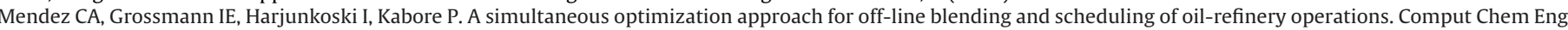
2006;30:614-34.

Moro LFL, Zanin AC, Pinto JM. A planning model for refinery diesel production. Comput Chem Eng 1998;22:S1039-42.

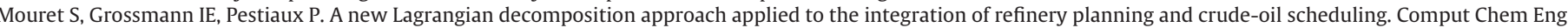
2011;35:2750-66.

Neiro SMS, Murata VV, Pinto JM. Hybrid time formulation for diesel blending and distribution scheduling. Ind Eng Chem Res 2014;53:17124-34.

Pinto JM, Joly M, Moro LFL. Planning and scheduling models for refinery operations. Comput Chem Eng 2000;24:2259-76.

Shah N, Saharidis GKD, Jia Z, Ierapetritou MG. Centralized-decentralized optimization for refinery scheduling. Comput Chem Eng 2009;33:2091-105.

Shah NK, Ierapetritou MG. Short-term scheduling of a large-scale oil-refinery operations: incorporating logistics details. AIChE J 2011;57:1570-84.

Shah NK, Ierapetritou MG. Integrated production planning and scheduling optimization of multisite, multiproduct process industry. Comput Chem Eng 2012;37:214-26.

Shah NK, Li Z, Ierapetritou MG. Petroleum refining operations: key issues, advances, and opportunities. Ind Eng Chem Res 2011;50:1161-70.

Tang L, Jiang S. The charge batching planning problem in steelmaking process using Lagrangian relaxation algorithm. Ind Eng Chem Res 2009;48:7780-7.

Wu D, Ierapetritou MG. Decomposition approaches for the efficient solution of short-term scheduling problems. Comput Chem Eng 2003;27:1261-76. 